\title{
Localization and Characterization of Gelsolin in Nervous Tissues: Gelsolin Is Specifically Enriched in Myelin-forming Cells
}

\author{
Junya Tanaka and Kenji Sobue \\ Department of Neurochemistry and Neuropharmacology, Biomedical Research Center, Osaka University Medical School, \\ Osaka 565, Japan
}

\begin{abstract}
Gelsolin is a $\mathrm{Ca}^{2+}$-sensitive actin filament-severing protein. To elucidate the role of gelsolin in nervous tissues, we have investigated localization and expression of gelsolin in rat CNS and PNS using biochemical and morphological methods with a polyclonal antibody against the $\mathrm{COOH}$-terminal fragment of plasma gelsolin. Immunohistochemical study showed that gelsolin was specifically enriched in oligodendrocytes and Schwann cells, and was also detected in myelin sheath, especially around the Ranvier's nodes. The immunohistochemical stainings using indirect immunofluorescence, avidin-biotin-peroxidase complex, and immunogold methods were carefully confirmed by immunoblotting against the tissue homogenates. The expressional changes of gelsolin in developing brain were investigated. The protein was detectable in newborn rat brain; however, it began to increase at 8-10 d after birth and reached maximal at 20-30 d when myelinogenesis actively occurred. After this period, the protein decreased gradually, although myelin basic protein was increasing until 6 months after birth. The immunostaining of gelsolin in Schwann cells was enhanced upon regeneration of injured sciatic nerves by freezing. Immunoelectron microscopy revealed that gelsolin was present not only in the cytoplasm but also in compact myelin. Following solubilization by detergents, gelsolin in the myelin fraction could be purified using anion exchange and blue Sepharose column chromatographies. The purified protein possessed a $\mathrm{Ca}^{2+}$-dependent severing activity against actin filaments similar to that of cytoplasmic and plasma gelsolin. These data strongly suggest that gelsolin in nervous tissues might be involved in lamellipodial movement to wrap axons of myelin-forming cells by modulating actin polymerization.
\end{abstract}

[Key words: gelsolin, oligodendrocyte, Schwann cell, myelination, remyelination, actin, cell motility]

Although mechanochemical enzymes such as myosins participate in many cellular motile events, the actomyosin system might not be the only molecular base of motile force for cell locomotion. For example, cells in which gene coding myosin II was disrupted are mobile (De Lozanne and Spudich, 1987:

Received Jan. 4, 1993; revised July 26, 1993; accepted July 29, 1993.

We thank Prof. Thomas P. Stossel, Massachusetts General Hospital and Har vard Medical School, for his critical discussion, and Dr. Takahiro Gotow, Department of Anatomy of Osaka University Medical School, for his important and kind advice. The present study was partly supported by grants from the Scientific Research Fund of the Ministry of Education, Science, and Culture of Japan.

Correspondence should be addressed to Kenji Sobue, Department of Neurochemistry and Neuropharmacology, Biomedical Research Center, Osaka University Medical School, 2-2 Yamadaoka, Suita, Osaka 565, Japan.

Copyright (C) 1994 Society for Neuroscience $0270-6474 / 94 / 141038-15 \$ 05.00 / 0$
Knecht and Loomis, 1987) and immunocytochemical studies have revealed that the moving lamellipodia do not contain myosin II (Symons and Mitchison, 1991). Instead, cell movement might be dependent on cycles of assembly and disassembly of actin filaments (Bray and White, 1988) regulated by many kinds of actin-modulating proteins (Stossel, 1989). Among these proteins, gelsolin was originally isolated from macrophages in rabbit lungs (Yin and Stossel, 1979). It severs and caps actin filaments in a $\mathrm{Ca}^{2+}$-dependent manner, and is involved in cell motility (Cunningham et al., 1991). Besides $\mathrm{Ca}^{2+}$, phosphatidylinositol-4-phosphate (PIP) and phosphatidylinositol-4,5bisphosphate $\left(\mathrm{PIP}_{2}\right)$ act as regulatory factors to dissociate the $\mathrm{Ca}^{2+}$-insensitive or EGTA-resistant binding between monomeric actin and gelsolin (Janmey and Stossel, 1987; Janmey et al., 1987). Judging from some reports on its localization in actively moving cells, gelsolin could be involved in many types of cell movement. In platelets, gelsolin might be involved in their shape changes during activation by thrombin (Hartwig et al. 1989; Hartwig, 1992). In cells transformed by Rous sarcoma virus, gelsolin concentrates in rosette contacts or podosomes (Gavazzi et al., 1989), which actively move on a time scale of minutes (Chen, 1989). Ruffling membrane, which also actively moves at the cell periphery, contains gelsolin (Cooper et al., 1988). These and other observations suggest gelsolin to be an essential factor for fast movement of cells.

In nervous tissue, active movement of many cell types is observed during synaptogenesis and myelinogenesis of developing brain, or in inflammatory reactions in the damaged brain. The filopodia of neuronal growth cones are well known to move rapidly in a $\mathrm{Ca}^{2+}$-dependent manner (Sobue and Kanda, 1989). Microglial cells might contain gelsolin, since they are regarded as the macrophages of the CNS. It is likely that gelsolin is involved in these neural cell activities, taking into account the above findings in non-neural cells. The presence of a gelsolinlike molecule with an $M_{r}$ of about $90 \mathrm{kDa}$ in brain in $1: 1$ complex form with actin has been reported (Nishida et al., 1981; Yin et al., 1981). Petrucci et al. (1983) have purified the gelsolin-like protein from nervous tissue possessing $\mathrm{Ca}^{2+}$-dependent severing activity against actin filaments. They also demonstrated the presence of this protein in cultured neurons. However, a second report on the neural gelsolin-like protein has denied its actin filament-severing activity and named it "Cap 90" (Isenberg et al., 1983). Since both authors used very severe conditions to dissociate the gelsolin-like protein from actin, the separated protein might have been denatured. Other investigators have reported that the $1: 1$ complex prepared from bovine brain severed tropomyosin-bounded actin filaments (Verkhovsky et al., 1984) or stress fibers of cultured fibroblasts (Verkhovsky et al., 
1987). Conflicting data on the localization of the gelsolin-like protein in nervous tissues have also been presented. It was not detected in neurons (except transient immunostaining of Purkinje cells) but in oligodendrocytes of immature rabbit brain (I egrand et al., 1986). Recently, the latter group has identified the protein in many cell types of developing rabbit retina including ganglion cells, Müller cells, and photoreceptors (Legrand et al., 1991). In addition, a gelsolin-like protein from adrenal medulla has been purified, but with a smaller molecular weight than that of macrophage gelsolin (Ashino et al., 1987; Rodriguez del Castillo et al., 1990; Sakurai et al., 1990).

In the present study, we have investigated the localization and characterization of the gelsolin-like protein in nervous tissues to resolve the aforementioned conflicting results and questions, such as which cells contain the gelsolin-like protein in nervous tissues, whether the protein severs actin filaments in the presence of $\mathrm{Ca}^{2+}$, and in which neural function the protein is involved. To achieve enhanced immunohistochemical staining, we prepared a specific antibody against the $\mathrm{COOH}$-tcrminal fragment of plasma gelsolin, containing the $\mathrm{Ca}^{2+}$-sensitive domain. The antibody specifically immunostained oligodendrocytes in brain and Schwann cells in peripheral nerves. Immunoreactivity was the strongest in rat brain at the period of 20$30 \mathrm{~d}$ after birth when myelinogenesis actively occurred. This morphological study was carefully confirmed by biochemical methods. The gelsolin-like protein was purified from a myelinenriched fraction. The purified protein had a severing activity against actin filaments similar to that of plasma gelsolin. We conclude that the protein is similar to plasma or macrophage gelsolin, and hypothesize that it regulates movement of lamellipodia of the myelin-forming cells to wrap around nerve fibers by regulating actin assembly.

\section{Materials and Methods}

Purification of human plasma gelsolin and its fragment. Human plasma gelsolin was purified by a combination of two methods as previously reported. One method was based on the $\mathrm{Ca}^{2+}$-dependent affinity of gelsolin to a DEAE-cellulose column (Kurokawa et al., 1990). Gelsolin was specifically eluted from the column in $\mathrm{C} \mathrm{Ca}^{2+}$-dependent manner. Another one was the specific elution of gelsolin from a blue Sepharose column with ATP (Yamamoto et al., 1989). Briefly, both methods werc employed as follows. Human plasma separated from fresh blood (1000 $\mathrm{ml}$ ) was fractionated between $35 \%$ and $50 \%$ ammonium sulfate saturation. After removing ammonium sulfate by dialysis, the fraction was applied to a DEAE-cellulose (DE-52, Whatman, Maidstone, England) column in the absence of $\mathrm{Ca}^{2+}$. Gelsolin was eluted with $1 \mathrm{mM} \mathrm{Ca}^{2+}$ Following dialysis to remove $\mathrm{Ca}^{2+}$, the eluted fraction was applied to a blue Sepharose (Pharmacia, Uppsala, Sweden) column. Gelsolin was eluted with 1 mm ATP (Sigma, St. Louis, MO). About $25 \mathrm{mg}$ of plasma gelsolin was obtained by this procedure.

To prepare specific antibody against the $\mathrm{COOH}$-terminal fragment of plasma gelsolin, a fast and easy method to separate the fragment from $\alpha$-chymotrypsin (Sigma)-digested gelsolin was developed. Proteolytic digestion was performed as previously reported (Kwiatkowski et al., 1985), and the three fragments $\left(\mathrm{NH}_{2}\right.$-terminal, central, and $\mathrm{COOH}-$ terminal fragments) were obtained. These digested fragments were applied to a blue Sepharose column equilibrated with Tris-EGTA buffer [10 mm Tris- $\mathrm{HCl}$, pH 8.0, $20 \mathrm{~mm} \mathrm{KCl}, 1$ mM EGTA, 0.5 mM dithiothreitol, $10 \mu \mathrm{M}$ (para-amidinophenyl)methanesulfonyl fluoride hydrochloride ( $p$-APMSF; Wako, Osaka, Japan)]. The $\mathrm{NII}_{2}$-terminal fragments were not adsorbed to the column, but the $\mathrm{COOH}$-terminal fragments were specifically eluted with Tris- $\mathrm{Ca}^{2+}$ buffer (the Tris-EGTA buffer containing $1.2 \mathrm{mM} \mathrm{CaCl}_{2}$ ) and the central fragments were recovered from the column with $1 \mathrm{M} \mathrm{NaCl}$ buffer (the Tris-EGTA buffer containing $1 \mathrm{M} \mathrm{NaCl}$ instead of $20 \mathrm{~mm} \mathrm{KCl}$ ).

Preparation of antibodies against human plasma gelsolin. Anti-human plasma gelsolin antiserum was raised in New Zealand White rabbits.
The antiserum was purified by an affinity column coupled with parent gelsolin, the $\mathrm{COOH}$-terminal fragment, or $\mathrm{NH}_{2}$-terminal fragment. The procedure for affinity purification of antibody has been described elsewhere (Sobue et al., 1988).

Immunoblotting to detect gelsolin in nervous tissues. For investigation on the distribution of gelsolin and its expressional change during development of rat nervous tissues, electrophoretic samples of tissue homogenates were prepared. Before taking out brains or sciatic nerves, rats anesthetized with ether were perfused with chilled saline through the heart. Blood was completely washed out to avoid contamination of plasma gelsolin into the homogenates. After removing the meninges, the brains were cut into several regions and weighed. Connective tissues around the sciatic nerves were carefully removed using a stereoscopic microscope. The tissues were minced and mixed with 10 or $20 \mathrm{vol}$ of homogenizing buffer ( $20 \mathrm{~mm}$ Tris- $\mathrm{HCl}, \mathrm{pH} 8.0,40 \mathrm{mM} \mathrm{NaCl}, 50 \mu \mathrm{M}$ $p$-APMSF, $10 \mu \mathrm{g} / \mathrm{ml}$ leupeptine) and homogenized using Polytron. In the study comparing the gelsolin content in the gray matter with that in the white, both tissues were punched out using glass capillaries. The homogenates were quickly dissolved in Laemmli's sample solution (Laemmli, 1970). The protein concentration was determined with BCA protein assay system (Pierce, Rockford, IL), and the serial samples for electrophoresis were adjusted to have equal protein concentrations. The samples were electrophoresed in the buffer system of Laemmli using SDS-polyacrylamide gel electrophoresis (SDS-PAGE). Immunoblotting was performed as described elsewhere (Tanaka et al., 1993). For the developmental study, immunostained bands were quantitated by densitometry.

Immunohistochemical staining. Morphological studies with the antigelsolin antibody (antibody against the $\mathrm{COOH}$-terminal fragment of gelsolin) were performed at both the light and electron microscopic level. In general, rats anesthetized with ether were fixed by transcardial perfusion for $30 \mathrm{~min}$ at room temperature with a fixative containing $4 \%$ paraformaldehyde in $0.1 \mathrm{~m}$ phosphate buffer ( $\mathrm{pH} 7.2)$. For immunoelectron microscopy, $0.1 \%$ glutaraldehyde was included in the fixative. For the developmental study (the results shown in Figs. 7, 8), the fixative contained $0.2 \%$ picric acid and $0.1 \%$ glutaraldehyde for better fixation of the immature brain. After fixation, the tissues were thin sectioned (20 $\mu \mathrm{m}$ thick) using a microslicer (DTK-1000, Dousaka E.M., Kyoto, Japan), and washed in Tris-buffered saline (TBS; $20 \mathrm{~mm}$ Tris-HCl, pH $7.6,150 \mathrm{~mm} \mathrm{NaCl}$ ). For immunoelectron microscopy with colloidal gold-labeled antibody, sections $5-10 \mu \mathrm{m}$ thick were prepared for better penetration of the antibody. Sciatic nerves were thin sectioned to $8 \mu \mathrm{m}$ thick using a cryostat. In the immunohistochemical study, we employed indirect immunofluorescence, avidin-biotin-peroxidase complex (EliteABC, Vector, Burlingame, CA) (Hsu et al., 1981), and immunogold methods. Although accumulation of biotin in oligodendrocytes has been reported (LeVine and Macklin, 1988), the avidin-biotin complex used in this study could not stain the cells unless both the anti-gelsolin antibody and the biotin-labeled secondary antibody were used (see Fig. $7 G$ ). Before immunoreaction, the sections were incubated in TBS containing $1 \%$ bovine serum albumin (BSA; crystallized and globulin free; Sigma). Following permeabilization with Triton X-100 (0.02-0.2\%) for 6-24 hr at $4^{\circ} \mathrm{C}$ or at room temperature, the sections were incubated in TBS containing the primary antibody and BSA (1\%) for $12-16 \mathrm{hr}$ at room temperature. After extensive washing, the sections were incubated in TBS containing fluorescent isothiocyanate (FITC)-labeled anti-rabbit IgG (2\%) (Sigma), rhodamine-labeled anti-mouse IgG (2\%) (Sigma), 5 $\mathrm{nm}$ colloidal gold-labeled anti-rabbit IgG (10\%) (E-Y Lab), or biotinlabeled anti-rabbit IgG $(0.3 \%)$ (Vector). When the ABC method was employed, the sections were incubated in $\mathrm{H}_{2} \mathrm{O}_{2}-$ methanol solution to destroy endogenous peroxidase activity, and then incubated in $\mathrm{ABC}$ solution $(1 \%)$. The immunoreaction was visualized using $3,3^{\prime}$-diaminobenzidine $(0.02 \%)$ (DAB; Dojin, Kumamoto, Japan) and hydrogen peroxide $(0.005 \%)$. Methods for double immunolabeling by indirect immunofluorescence have been described in detail elsewhere (Tanaka et al., 1993). Zeiss axiophot was used for light microscopic observation. For electron microscopy, the immunostained samples using the $\mathrm{ABC}$ or immunogold methods were postfixed with $1 \% \mathrm{OsO}_{4}$, dehydrated in graded ethanols, flat embedded in Luveak 812 (Nakarai, Kyoto, Japan), and ultrathin sectioned. The sections were stained with lead citrate and examined in a JEM 100CX electron microscope (JEOL, Tokyo, Japan) operated at $100 \mathrm{kV}$.

Nerve injury and regeneration. To investigate the role of gelsolin in peripheral nerves, the regenerating experiment was performed according to the method of Tashiro and Komiya (1991). After anesthesia with 


\section{$a b c d e f g a b c d e$}
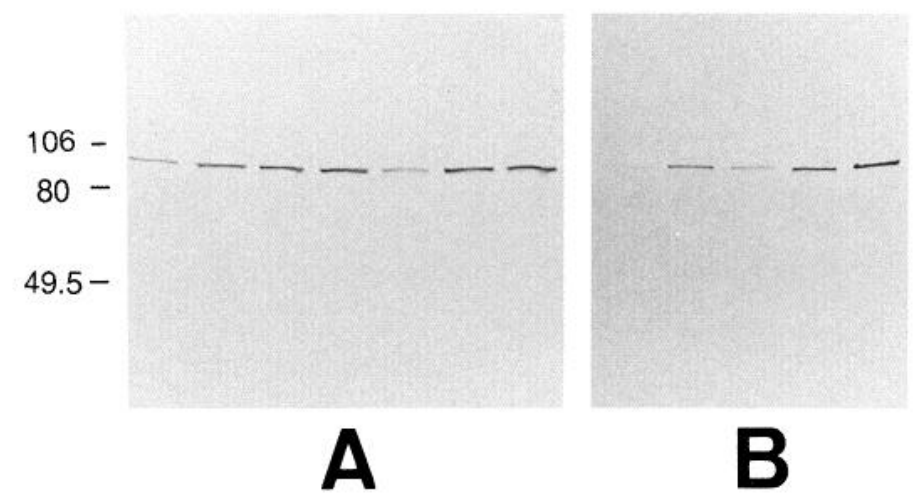
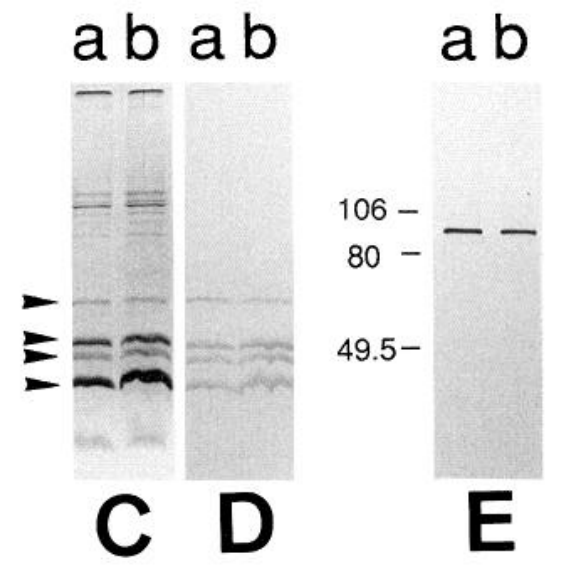

Figure 1. Distribution of gelsolin in nervous tissues of 8 -week-old male rats was examined by immunoblotting with the anti-gelsolin antibody. The homogenates of the endbrain, the interbrain, the midbrain, the pons, the cerebellum, the medulla oblongata, and the spinal cord were applied on lanes $a-g$, respectively, of $A$. Fourteen micrograms of protein were loaded on each lane. Gelsolin predominantly distributed in inferior regions of the CNS. The contents of gelsolin in brain were compared between the gray and the white matter $(B)$. Lanes $a-d$ indicate the cerebral cortex, the corpus callosum, the hippocampus, and the middle cerebellar peduncle, respectively. The homogenate of the sciatic nerves was loaded on lane $e$ of $B$. Seven micrograms of protein were applied on lanes $a, b, c$, and $d$ and $15 \mu \mathrm{g}$ on lane $e$ of $B$. Note that gelsolin was predominantly detected in the white matter (lanes $b$ and $d$ ). The protein was also present in the peripheral nerves. A 9\% polyacrylamide gel was used for $A$ and $B$. Lanes $a$ and $b$ in $C-E$ show the myelin fraction prepared from 30-d- and 13-week-old rat brains, respectively. $C$ shows the Coomasie brilliant blue-stained gel $(15 \%)$, and the four arrowheads indicate MBP that was confirmed by immunoblotting using the anti-MBP antibody $(D)$. $E(9 \%$ gel) shows immunoblotting by the anti-gelsolin antibody. Numbers indicate $M,(\mathrm{kDa})$.

ether and pentobarbital, the sciatic nerves of rats ( 8 weeks old) were frozen by pressing with a stainless steel stick precooled in liquid nitrogen. At $5 \mathrm{~d}$ after the operation, the injured nerves were taken out and fixed in phosphate buffer containing $4 \%$ paraformaldehyde for $3 \mathrm{~d}$ at $4^{\circ} \mathrm{C}$. After fixation, the nerves were thin sectioned by cryostat and immunostained using the $\mathrm{ABC}$ method as described above.

Preparation of myelin fraction and solubilization of brain gelsolin. The myelin fraction was prepared as described by Norton and Poduslo (1973), with slight modification. In solubilization experiments of gelsolin from the myelin fraction, we referred to a report on solubilizing myelin membrane (Aveldaño et al., 1991) and tried Triton X-100, Lubrol PX (Sigma), $n$-octyl- $\beta$-D-thioglucoside (Dojin), CHAPS (Dojin), and deoxycholic acid. Among the detergents, $1 \%$ Lubrol PX and deoxycholic acid solubilized most of gelsolin in the myelin fraction (data not shown). Brain gelsolin was also solubilized from the myelin fraction or the crude myelinated axon fraction prepared by the method of Liem et al. (1978).

Falling ball viscometry and electron microscopy on negatively stained specimens. To characterize the purified brain gelsolin, its effect on skeletal muscle actin was evaluated by falling ball viscometry and electron microscopic observation. The methods were described elsewhere (Ashino et al., 1987). The final assay conditions for both methods were as follows: brain gelsolin $(4 \mu \mathrm{g} / \mathrm{ml})$; actin filaments $(1.2 \mathrm{mg} / \mathrm{ml})$ or monomeric actin $(0.7 \mathrm{mg} / \mathrm{ml})$; in $20 \mathrm{~mm}$ PIPES, pH 7.0, $100 \mathrm{~mm} \mathrm{KCl}, 2$ $\mathrm{mm} \mathrm{MgCl}_{2}, 1 \mathrm{~mm}$ dithiothreitol, $1 \mathrm{~mm}$ EGTA, $1 \mathrm{~mm} \mathrm{ATP}$, and $10 \mu \mathrm{M}$ $p$-APMSF. When the effects of gelsolin in the presence of $\mathrm{Ca}^{2+}$ were observed, $1.2 \mathrm{~mm} \mathrm{CaCl}_{2}$ was included in the assay mixture. Following incubation at $23^{\circ} \mathrm{C}$ for $30 \mathrm{~min}$, the apparent viscosity of the mixtures was measured. The mixtures were also negatively stained with $2 \%$ uranyl acetate solution and viewed by JEOL $100 \mathrm{CX}$ electron microscopy.

Other materials. Antibodies against myelin basic proteins (polyclonal) (MBPs), glial fibrillary acidic protein (GFAP) (polyclonal), and neurofilament $68 \mathrm{kDa}$ protein (monoclonal) were purchased from Zymed (San Francisco, CA), Biomeda (Foster City, CA), and BioMakor (Rehoot, Israel), respectively. Rhodamine-labeled phalloidin for fluorescent staining of actin filaments was obtained from Sigma. Normal rabbit IgG for the control study was from Jackson ImmunoResearch Lab (West Grove, PA). Actin was purified from rabbit skeletal muscle (Pardee and Spudich, 1982). Molecular weight markers were obtained from Bio-Rad (Richmond, CA).

\section{Results}

Preparation of antibody against the $\mathrm{COOH}$-terminal fragment of plasma gelsolin

We first purified an antibody against the parent molecule of plasma gelsolin using the affinity column coupled with the purified protein. This antibody, however, cross-reacted with some proteins other than the $90 \mathrm{kDa}$ band, or gelsolin in the rat brain homogenate (data not shown). Plasma gelsolin has an additional sequence in the $\mathrm{NH}_{2}$-terminus that is absent in the cytoplasmic gelsolin (Kwiatkowski et al., 1986). The COOH-terminal fragment possesses $\mathrm{Ca}^{2+}$-dependent actin-binding domain (Kwiatkowski et al., 1985), and is common between plasma and cytoplasmic gelsolins (Kwiatkowski et al., 1986). Therefore, we prepared the anti-COOH-terminal fragment-specific antibody from anti-gelsolin antiserum and the antibody was specifically cross-reacted with gelsolin in nervous tissues, as shown in Figure 1. By contrast, the anti- $\mathrm{NH}_{2}$-terminal fragment antibody preferentially cross-reacted with other proteins (data not shown). In the following studies, we used the antibody against the $\mathrm{COOH}$ terminal fragment of gelsolin as anti-gelsolin antibody.

\section{Distribution of gelsolin in nervous tissues-immunoblotting study}

The distribution of the protein in nervous tissues was investigated by immunoblotting using the anti-gelsolin antibody. Although gelsolin is distributed throughout the CNS, the protein was more enriched in the inferior regions of the brain (the pons, the medulla oblongata, and the spinal cord) than in the cerebrum and the cerebellum (Fig. $1 A$ ). Figure $1 B$ shows that gelsolin was predominantly present in the white matter as opposed to the gray matter (compare the immunoreaction between lanes a, $c$ and $b, d$; the former are the gray matter, and the latter, the white). Gelsolin was also detected in the homogenate of rat sciatic nerves (Fig. 1B, lane e). We further examined its localization in the myelin fraction, which was prepared according to the method of Norton and Poduslo (1973). Figure $1 C$ shows electrophoretogram of the myelin fraction. The arrowheads indicate the four MBPs that were confirmed by the antibody against MBP (Fig. 1D). Corresponding immunoblots by the anti-gelsolin antibody are shown in Figure $1 E$. Gelsolin was present in the myelin fractions. 

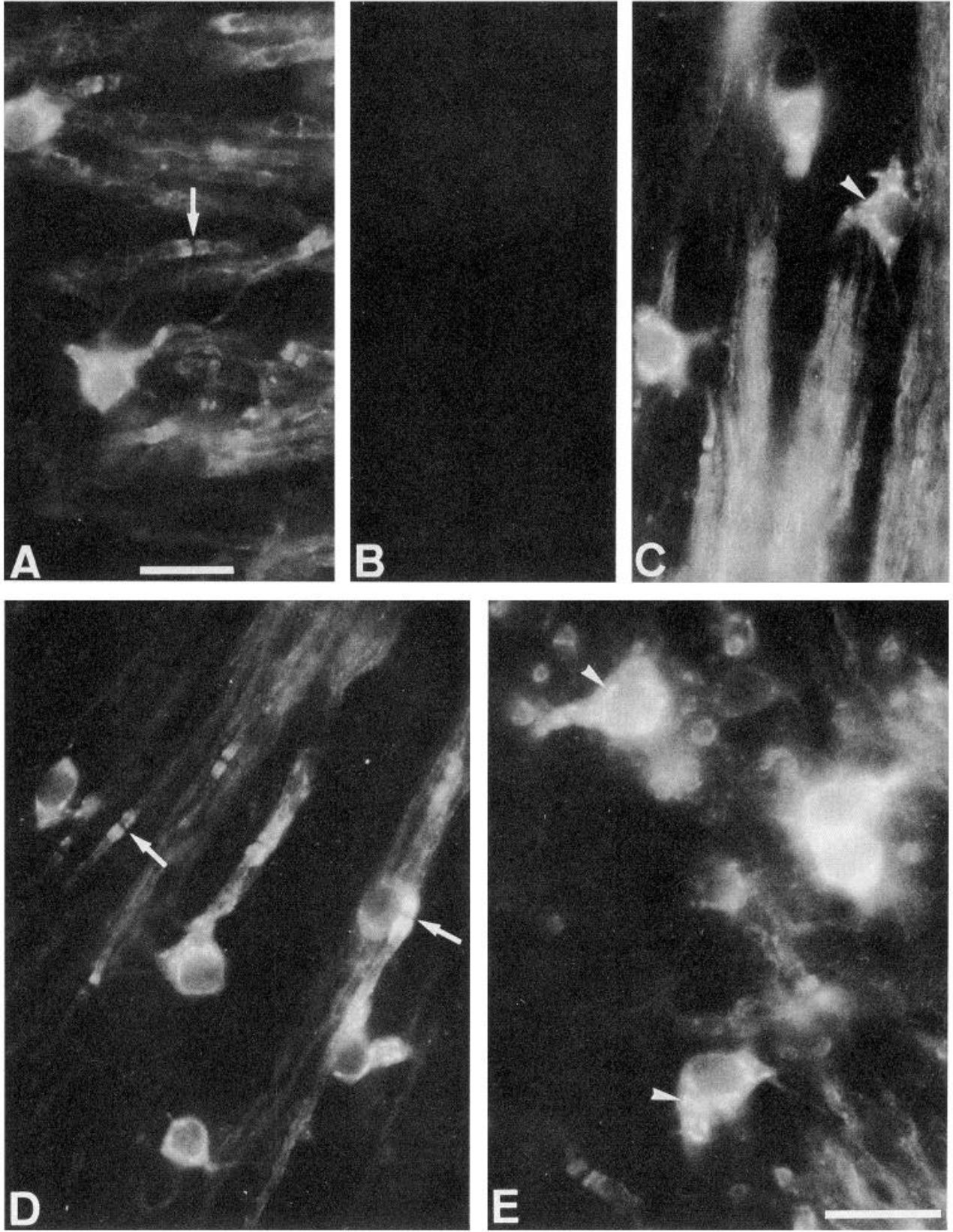

Figure 2. Oligodendrocytes in the pons of 15-d-old rats were positively immunostained using indirect immunofluorescence with the anti-gelsolin antibody $(A, C-E)$. $A$ shows the cells in the region of facial nerve nucleus projecting their processes to thick axons of the primary motoneurons, which were identified by double immunolabeling with the anti-neurofilament antibody (data not shown). $B$ shows the control staining with normal rabbit IgG in the same brain region as $A$, in which no significant staining is observed. Protein concentration of diluted normal rabbit $\mathrm{IgG}$ was adjusted to be equal to that of the anti-gelsolin antibody solution $(0.2$ $\mu \mathrm{g} / \mathrm{ml})$. The cells in the medial cerebellar peduncle are shown in $C . D$ and $E$ show the reticular formation in the pons. Note the staining of myelin sheath as well as cell bodies of oligodendrocytes. Myelin sheath at the Ranvier's nodes was remarkably stained (arrows in $A$ and $D$ ). Arrowheads in $C$ and $E$ indicate the patchy immunostaining in the cytoplasm of the cells. Transverse section of myelin sheath is shown in $E$. Gelsolin immunoreactivity distributed around the axons. Scale bars, $20 \mu \mathrm{m}$ (same scale in $A-D$ ).

\section{Immunohistochemical staining of gelsolin in oligodendrocytes}

To identify gelsolin-containing cells in rat brain, indirect immunofluorescence method was used with the anti-gelsolin antibody. Figure 2 shows the immunoreactive cells in the pons from 15-d-old rats. The cells have the characteristic morphology of oligodendrocytes, having few processes without any branching and a small cell body (about $20 \mu \mathrm{m}$ ). Note the enriched staining of myelin sheath, especially of Ranvier's nodes (arrows in Fig. $2 A, D$ ). Thick myelinated axons from the primary motoneurons in the facial nerve nucleus (Fig. $2 A$ ) and thin axons of the middle cerebellar peduncle (Fig. $2 C$ ) were both well stained. Ring-like staining could be seen in the transverse section of myelinated neurites (Fig. 2E). In the cell body, the cytoplasm staining was patchy (Fig. 2, arrowheads). The highly stained regions corresponded to Golgi complex, which was identified by immunoelectron microscopy (see Fig. 10). Normal rabbit
IgG did not stain any specific structures (Fig. $2 B$ ). Figure $3, A$ and $B$, shows the same field in the pons of the 21 -d-old rat. The location of neuronal cell bodies and neurites was identified using monoclonal antibody against neurofilament $68 \mathrm{kDa}$ protein (Fig. $3 A$ ). The positions of the neurons identified in Figure $3 A$ are indicated in Figure $3 B$ by asterisks. These micrographs show that gelsolin was below the detectable level in neurons. Next, we identified the location of astrocytes using anti-GFAP antibody. In Figure 3, $C$ and $D$ are the same field of the cerebellar white matter. Since both the anti-GFAP and the anti-gelsolin antibodies had been produced in rabbits, we performed double labeling using the combination of the $\mathrm{ABC}$ and indirect immunofluorescence methods. The $\mathrm{ABC}$ (Elite-ABC) method is 100 times more sensitive than indirect immunofluorescence. Therefore, after immunostaining by the $\mathrm{ABC}$ method with a very low concentration of the anti-gelsolin antibody, the brain section can be immediately immunostained with the anti-GFAP 
Figure 3. The specific enrichment of gelsolin in oligodendrocytes was confirmed by a double-immunolabeling study. Antibodies against neurofilament $68 \mathrm{kDa}$ protein $(A)$, GFAP $(C)$, and gelsolin $(B, D, F)$ were used, and rhodamine-phalloidin staining is shown in $E$. $A$ and $B$, and $C$ and $D$ show the same field of the pons (the reticular formation) and the cerebellar white matter, respectively. $A$ shows the neuronal cell bodies and neurites, which were not stained with the anti-gelsolin antibody (B). Asterisks in B indicate the positions of two neurons corresponding to those marked in $A$. Astrocytes stained with the anti-GFAP antibody are seen in $C$. The anti-gelsolin antibody did not recognize astrocytes $(D)$. Two asterisks in $D$ show the positions of astroytes indicated by arrowheads in $C . E$ and $F$ are the same field of the pons. The fluorescence of rhodamine-labeled phalloidin shows the distribution of actin filaments $(E) . F$ shows the gelsolin-containing oligodendrocytes (large arrows). The cell bodies of oligodendrocytes were not stained with rhodamine-labeled phalloidin, but the processes and the myelin sheaths were stained (small arrows and arrowheads, respectively). The cells, which do not contain both actin filaments and gelsolin, may be astrocytes (asterisks in $E$ and $F$ ). The brain section shown in this figure was prepared from 21-d-old rat. Scale bar, 30 $\mu \mathrm{m}$.
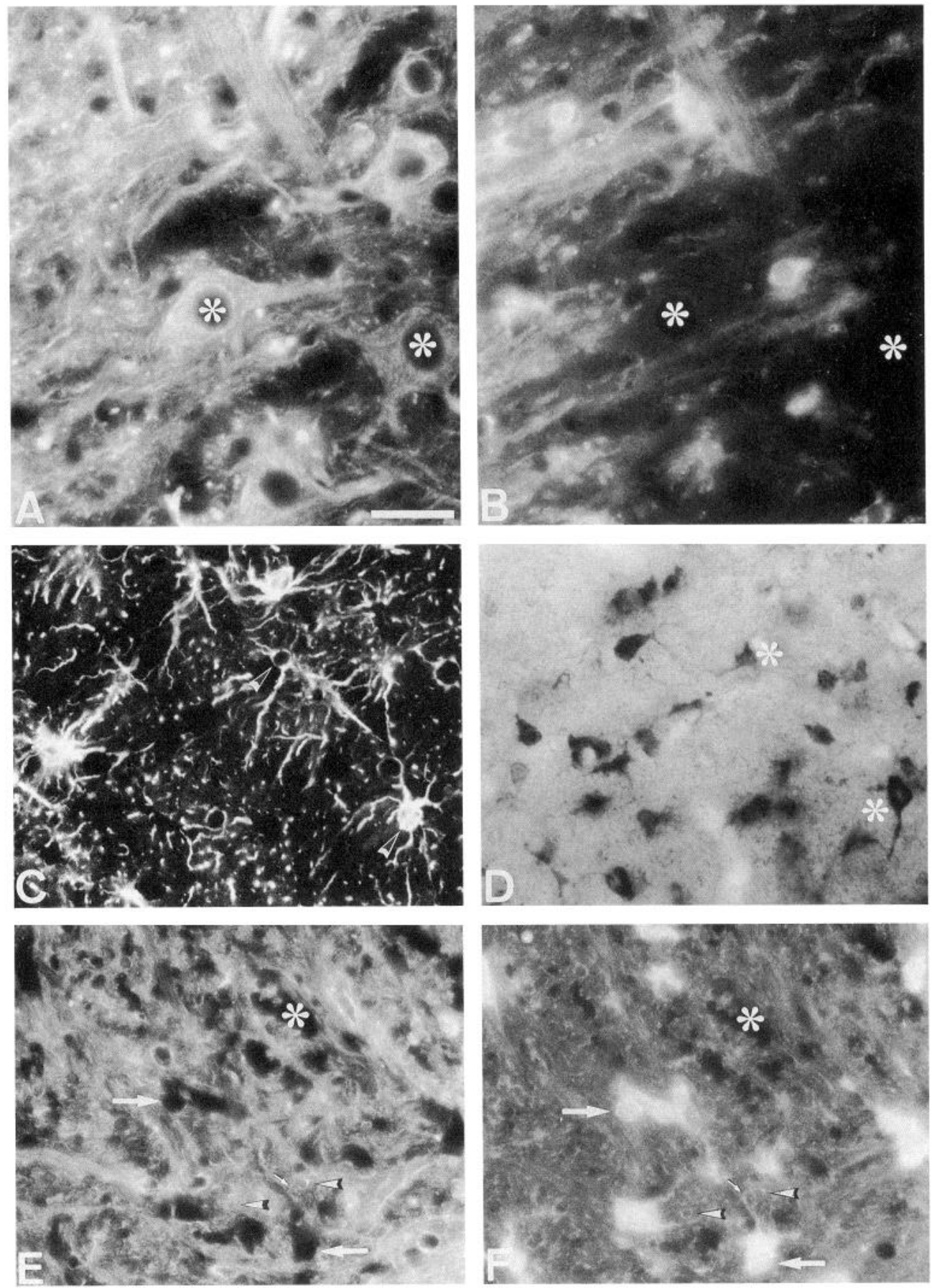

antibody by the immunofluorescence method without washing out the former antibody. By comparing Figure 3, $C$ and $D$, the absence of detectable gelsolin in astrocytes is clear. The specific enrichment of gelsolin in oligodendrocytes is compatible with the results of the immunoblotting study shown in Figure 1.

Figure 3, $E$ and $F$, shows the same field of the pons stained by rhodamine-labeled phalloidin, to detect actin filaments $(E)$, and by the anti-gelsolin antibody $(F)$. Note the lack of rhodamine fluorescence in the cell bodies of oligodendrocyte $(E$, arrows), which are stained with the anti-gelsolin antibody $(F)$. Alternatively, the processes and the myelin sheath from oligodendrocytes seemed to contain actin filaments. Such distribution of actin filaments is compatible with the previous obser- vations on cultured oligodendrocytes (Kachar et al., 1986). The cells indicated by asterisks in Figure $3, E$ and $F$, might be astrocytes, which did not contain detectable actin filaments or gelsolin.

\section{Localization of gelsolin in peripheral nerves}

Gelsolin was localized in thin cytoplasm of Schwann cells in rat sciatic nerves with the enhanced $\mathrm{ABC}$ method using nickel ion (Fig. 4A). Significant immunostaining was not observed when normal rabbit IgG was used instead of the anti-gelsolin antibody (Fig. $4 B$ ). As seen in the brain, regions near Ranvier's nodes were densely immunostained by anti-gelsolin antibody (Fig. $4 \mathrm{~A}$, arrowheads). In addition, the antibody also immunostained 

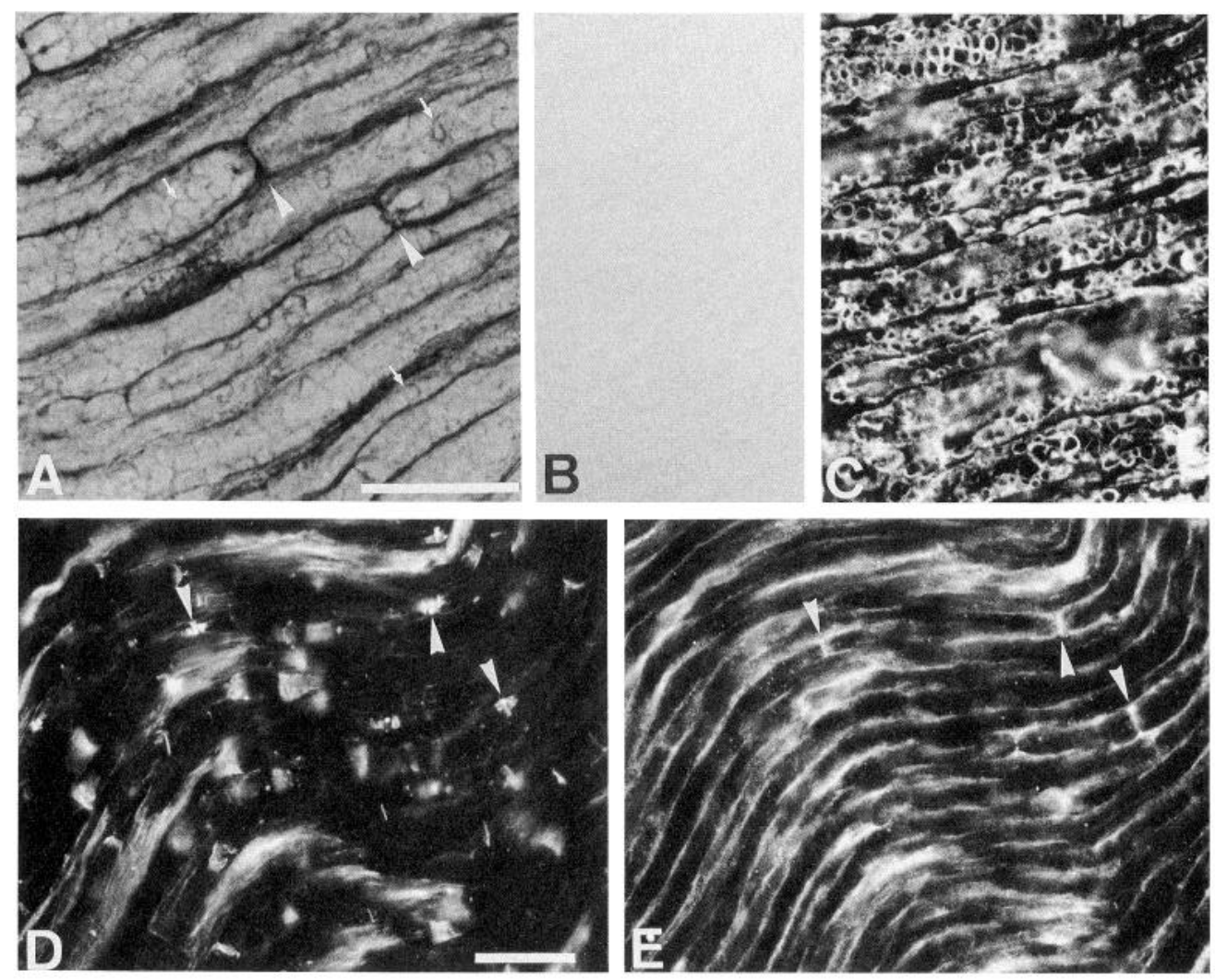

Figure 4. Localization of gelsolin (A and $E$ ), MBP $(C)$, and actin filaments $(D)$ in the sciatic nerves of 8-week-old male rats. The immunoreaction was developed using the enhanced $\mathrm{ABC}$ method with nickel ion $(A$ and $B)$. Indirect immunofluorescence was employed in $C$ and $E$. Gelsolin was localized in both the cytoplasm of Schwann cells and "neurokeratin," or myelin sheath (arrows in A). Gelsolin immunoreactivity was strong around the Ranvier's nodes (arrowheads in A). No specific immunostaining was observed with normal rabbit IgG $(B)$. MBP was localized in the neurokeratin of myelin sheath-like gelsolin $(C) . D$ and $E$ show the same field of the sciatic nerve stained with rhodamine-labeled phalloidin $(D)$ and the anti-gelsolin antibody $(E)$. Actin filaments were scarcely localized in the cytoplasm except the region around the nodes (arrowheads in $D$ ). Scale bars, 30 $\mu \mathrm{m}(A-C$, and $D$ and $E$ are the same scale, respectively). bubble-like structures (Fig. $4 A$, arrows). These structures are "neurokeratins," which are composed of proteins in the myelin sheath of peripheral nerves (Adams et al., 1971). MBP was specifically localized in the neurokeratin (Fig. 4C). Therefore, gelsolin was localized in the myelin sheath of both CNS and PNS. Figure $4, D$ and $E$, indicates the same field of the sciatic nerve double stained with rhodamine-labeled phalloidin $(D)$ and the anti-gelsolin antibody $(E)$. In Figure $4 E$, the neurokeratin was not seen, probably due to a lower sensitivity of the indirect immunofluorescence method. As in the CNS, actin filaments were scarce in the cytoplasm of Schwann cells, although they were accumulated in the regions near the nodes (Fig. $4 D$, arrowheads).

\section{Changes of gelsolin in developing brain}

Changes in gelsolin content in developing rat brain were examined in the forebrain (the end and the interbrain) and the brainstem (the midbrain, the pons, and the medulla oblongata). Twelve brain homogenates were prepared from rats $1 \mathrm{~d}$ to 29 weeks old, of which protein concentrations were adjusted to be equal. The same volumes of homogenates were electrophoresed and immunoblotted by both the anti-gelsolin and the anti-MBP antibodies. Figure $5 A$ shows the immunoblots by the anti-gelsolin antibody. Immunoreacted bands with $90 \mathrm{kDa}$ were quantitated by densitometry, as shown in Figure $5 B$. Gelsolin was slightly detectable in 1-d-old rat brain, and began to increase at $8 \mathrm{~d}$ after birth in the brainstem and $10 \mathrm{~d}$ in the forebrain. The amount of gelsolin always altered earlier in the brainstem than in the forebrain. When gelsolin content began to increase, MBP simultaneously became detectable. The expression of gelsolin reached maximal $21 \mathrm{~d}$ after birth in the brainstem and $28 \mathrm{~d}$ in the forebrain, after which gelsolin content decreased gradually.
In contrast to the expressional changes of gelsolin, MBP increased at nearly a constant rate until 29 weeks after birth.

These expressional changes of gelsolin and MBP were morphologically investigated. Forebrain sections were prepared from rats aged $3,8,15$, and $21 \mathrm{~d}$ and $4,8,16$, and 24 weeks, and immunostained by the anti-gelsolin and the anti-MBP antibodies (Fig. 6). Gelsolin-positive oligodendrocytes were clearly observed in the pons of 8-d-old rat brain (data not shown), but only small numbers of the gelsolin-positive cells were identified in the endbrain. Numbers of oligodendrocytes reacting to the anti-gelsolin antibody in the forebrain increased dramatically at $15 \mathrm{~d}$ after birth and were maximal at 4 weeks after birth. Thereafter, the gelsolin immunoreactivity decreased, though MBP was easily detectable. These immunohistochemical results are compatible with those of the immunoblotting study shown in Figure 5. Figure 7 also shows the gelsolin immunostaining of oligodendrocytes in developing brains. The immunoreaction at the myelin sheath was most evident in the 15-d-old rat brain (Fig. 7A), and the cell bodies were stained the strongest at 21$28 \mathrm{~d}$ after birth (Fig. $7 B, C$ ). Although the size of oligodendrocytes decreases with age in rats (Raine, 1989), they could be identified using the anti-gelsolin antibody even in the brain of a 6-month-old rat (Fig. $7 F$ ). Normal rabbit IgG did not significantly stain any structures (Fig. $7 G$ ).

\section{Alteration of gelsolin immunoreactivity in Schwann cells accompanying nerve regeneration}

The changes in gelsolin immunoreactivity of injured rat sciatic nerves were investigated. Damaged sciatic nerves were immunostained with the anti-gelsolin antibody using the $\mathrm{ABC}$ method. The upper panel of Figure 8 shows regenerating sciatic nerve. The arrow indicates where the nerve was damaged. Proximal 
A

$\begin{array}{llllllllllllllllllllllll}1 & 3 & 5 & 8 & 10 & 13 & 15 & 21 & 28 & 8 w & 16 w & 29 w & 1 & 3 & 5 & 8 & 10 & 13 & 15 & 21 & 28 & 8 w & 16 w & 29 w\end{array}$

106 -

80 -

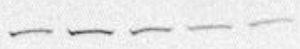

49.5 -

Forebrain

Brain stem

B

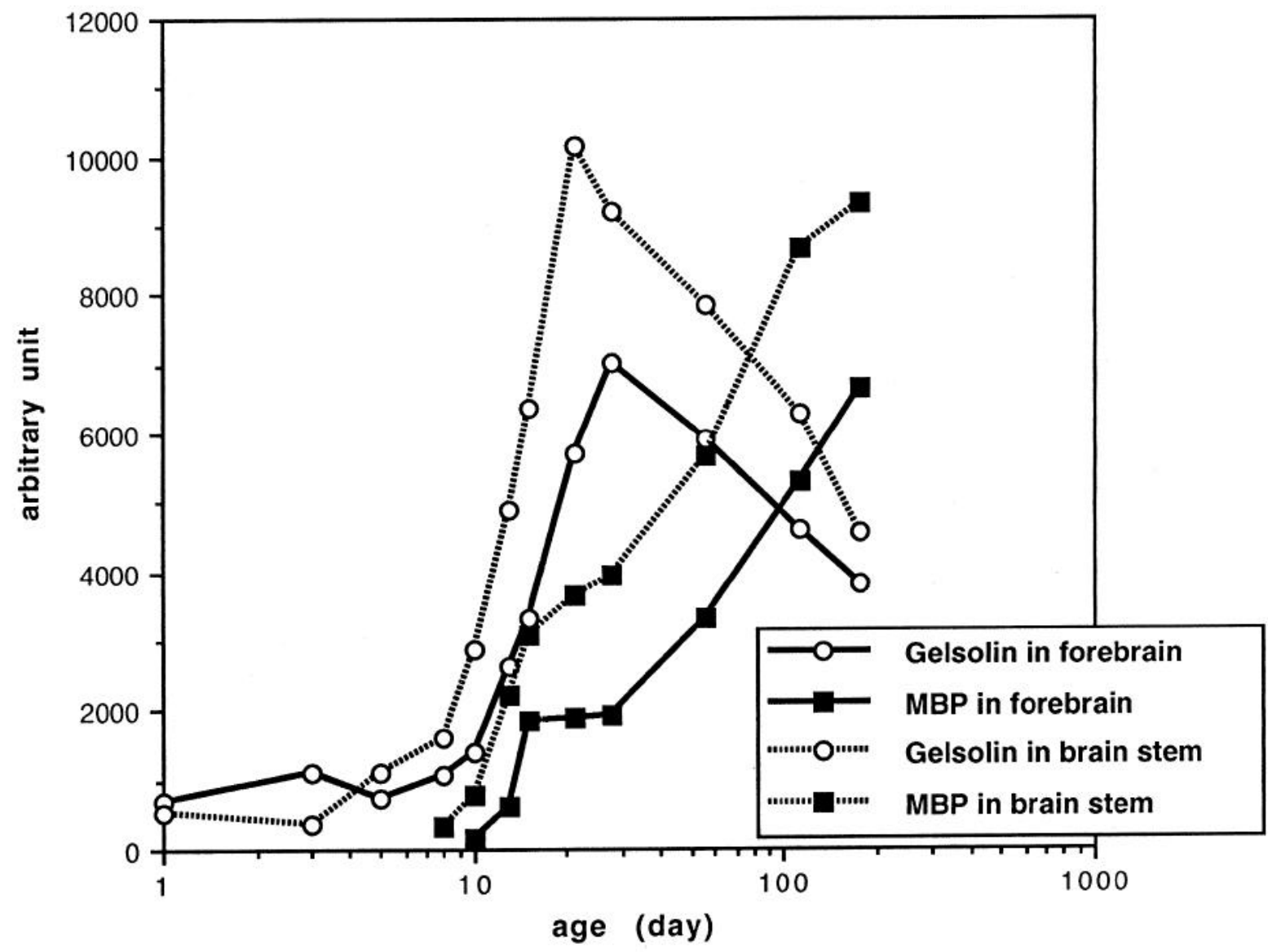

Figure 5. The developmental changes of gelsolin in the forebrain (the end- and interbrain) and the brainstem (the midbrain, the pons, and the medulla oblongata). Following preparation of 12 brain homogenates, the homogenates were immunoblotted with antibodies against gelsolin and MBP. The results of immunoblotting for gelsolin are shown in A. The numbers over the blots indicate the ages (day or week) of rats. The numbers on the left show $M_{r}(\mathrm{kDa})$. Slight cross-reaction with another band is detected in the early stage of forebrain development. The gelsolin bands were densitometried and the values are plotted along the y-axis against the rat age (day: the $\mathrm{x}$-axis) $(B)$. Only a small amount of gelsolin was detected in newborn rat brain, but the amounts dramatically increased 8-10 d after birth. MBP began to be detectable at the same period and continued to increase until six months. By contrast, the amount of gelsolin reached maximal at $20-30 \mathrm{~d}$ and decreased thereafter. 
MBP
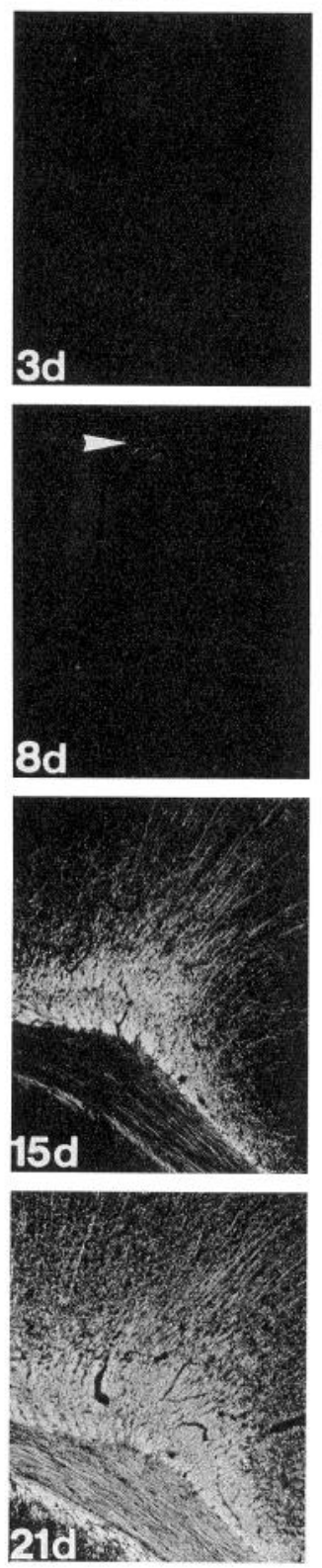

\section{Gelsolin}
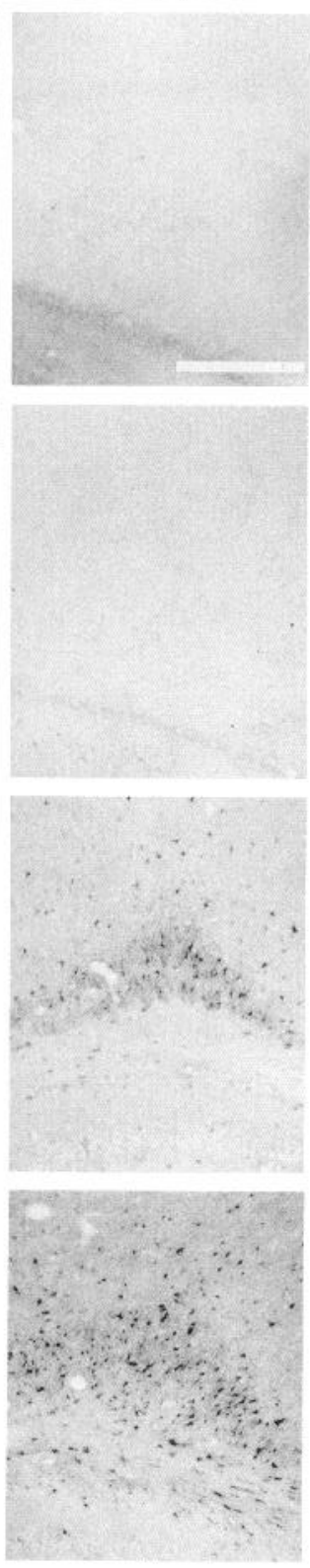

MBP
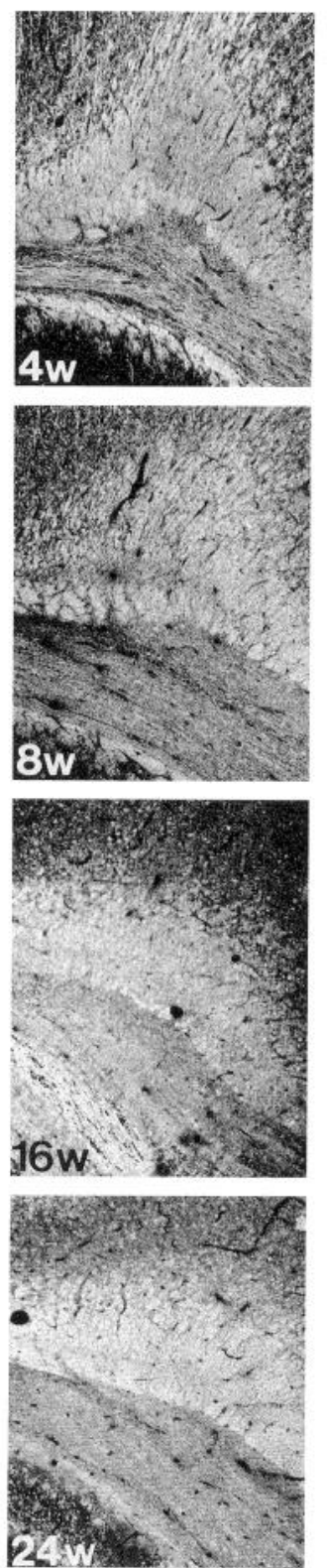

\section{Gelsolin}
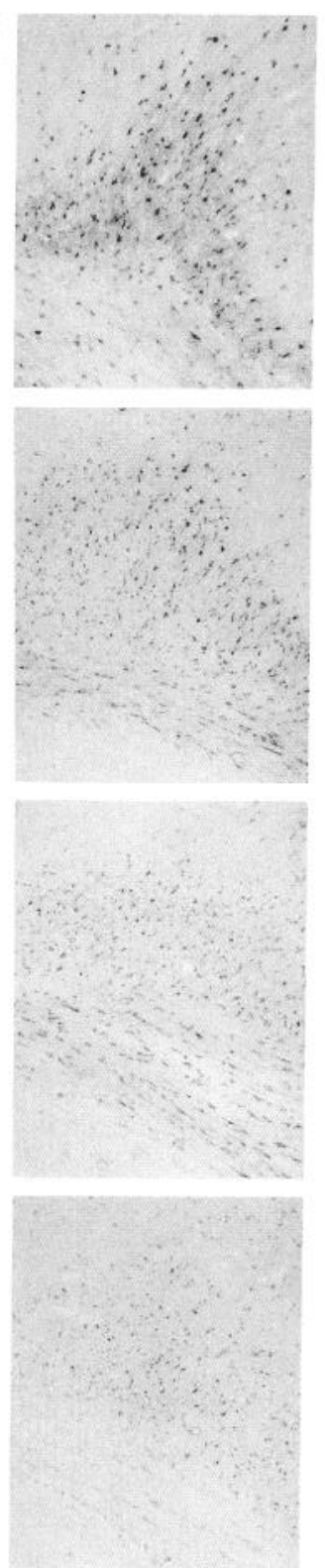

Figure 6. The developing forebrains (including the right parietal lobe, the corpus callosum, and the hippocampus) were immunostained with the antigelsolin and anti-MBP antibodies. Indirect immunofluorescence and $\mathrm{ABC}$ methods were employed to detect MBP and gelsolin, respectively. The fixed brains were prepared from rats $3,8,15$, and $21 \mathrm{~d}$ and $4,8,16$, and 24 weeks old, as shown in the photomicrographs. An arrowhead in photomicrograph of 8 -d-old rat brain indicates myelinated axons fluorescently labeled. Scale bar, $500 \mu \mathrm{m}$. to the damage (left side of panel), the gelsolin immunoreaction was moderate. Immunoreaction increased to maximum around the damage point, and again became weaker distal to this point. In Figure 81, slightly enhanced immunoreaction of gelsolin and some gelsolin-positive macrophages (arrowheads) are also observed. Figure 82 shows mostly short Schwann cells, which appear during nerve regeneration. The anti-gelsolin antibody stained these Schwann cells darker than normal cells. In the distal region, the destroyed myelinated axons predominate and the immunoreaction for gelsolin was weak (Fig. 83). In the regenerating region, actin staining by rhodamine-phalloidin was also enhanced (data not shown). Normal rabbit IgG did not stain Schwann cells (Control).

\section{Localization of gelsolin in oligodendrocytes at electron microscopic level}

The localization of gelsolin in oligodendrocytes was further investigated by immunoelectron microscopy. Brains (pons) from 21-d-old rats were thin sectioned and immunostained using the ABC method before embedding. Figure $9 A$ shows an interfascicular cell, a type of oligodendrocyte actively forming myelin sheath (Raine, 1989), surrounded by many myelinated axons. Besides dense staining of the cell body, the myelin sheaths were also gelsolin positive, which confirms the results shown in Figure $1 C-E$. Significant staining was not observed in the control experiment (Fig. 9B). Figure $9 C$ shows two perineuronal satellite cells attaching to neurons (labeled "N"). The satellite cell is another type of oligodendrocyte, which presumably supports the neuronal metabolism (Raine, 1989). Although the satellite cells were also gelsolin positive, as a rule the immunoreaction was not as strong as that of interfascicular cells. The positive cells in Figure 9 do not possess the features of microglial cells. Although glutaraldehyde-containing fixatives caused considerable nuclear staining as shown here, oligodendrocyte nuclei were not stained in the brain fixed only with paraformaldehyde (see Fig. 2). 
Figure 7. Oligodendrocytes in the developing forebrains immunostained with the anti-gelsolin antibody. $A-F$ correspond to the bottom area of the right parietal lobes of brains from rats 15,21 , and $28 \mathrm{~d}$ and 8,16 , and 24 weeks old, respectively. Normal rabbit $\mathrm{IgG}$ does not stain oligodendrocytes $(G)$ in 28 -d-old rat brain. The immunoreaction in myelin sheath was the most evident in 15-d-old rat brain, after which the immunoreaction grows weaker with age. By contrast, oligodendrocyte cell bodies were immunostained, though they become smaller with age. Scale bar, $100 \mu \mathrm{m}$.
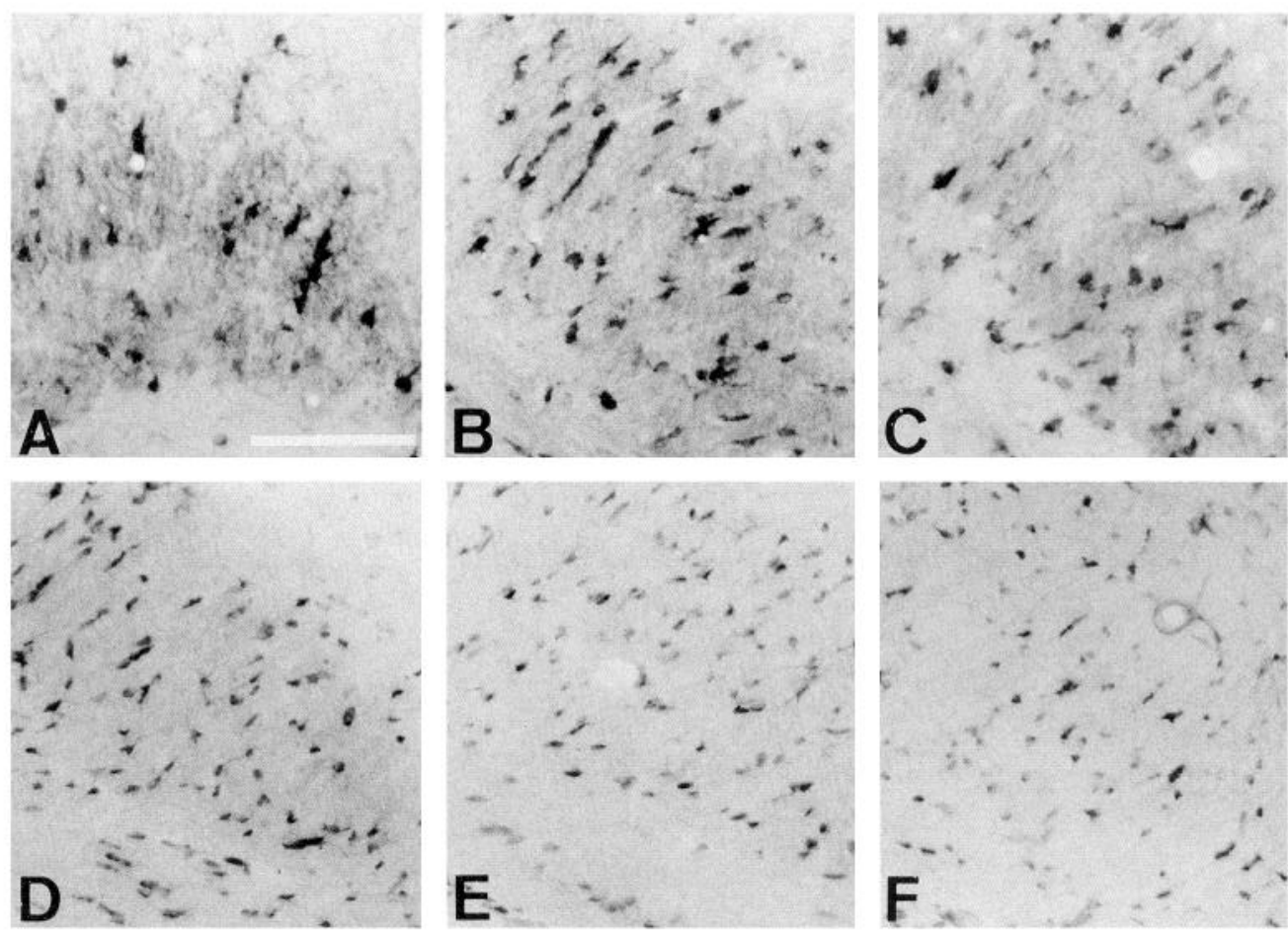

Figure $10 \mathrm{~A}$ shows a region where an oligodendrocyte projects a process that is wrapping an axon. The transitional region of the process was gelsolin positive, as was the myelin sheath itself. Figure $10 B$ shows the density of immunostaining around the Golgi apparatus. Therefore, the patchy stainings shown in Figure 2 are due to the accumulation of cytoplasmic gelsolin around the Golgi apparatus.

The localization of gelsolin in the myelin sheaths was intimately observed by immunoelectron microscopy using antirabbit IgG antibody labeled with colloidal gold particles. The particles were observed on the compact myelin (Fig. 11A). Normal rabbit IgG did not stain any structures (Fig. 11B).

\section{Purification and characterization of brain gelsolin}

We have purified and partially characterized the gelsolin in the myelin fraction to elucidate whether it has the severing and capping activity against actin similar to that of cytoplasmic or plasma gelsolin. At first, the solubility of myelin gelsolin was examined using various detergents. The lower concentration of the detergents such as $0.1 \%$ Triton X-100 or deoxycholic acid solubilized only a small portion of gelsolin and MBP (data not shown). The higher concentration ( $1 \%)$ of deoxycholic acid, however, could solubilize most of the gelsolin in the myelin fraction.

Gelsolin was purified from the myelin fraction prepared by the axonal flotation method (Fig. 12). The crude fraction of myelinated axons prepared from 30 -d-old rat brains ( $42 \mathrm{gm}$ ) was washed in water several times to remove the contents of the axons. The myelin fraction is shown in lane a of Figure $12 \mathrm{~A}$ (silver-stained gel), and Figure $12 B$ is immunoblotting for gelsolin. Proteins solubilized by deoxycholic acid are shown in lane b. After removing the detergent by dialysis, many insoluble substances appeared, but gelsolin was not precipitated by centrifugation (lane c). The dialyzed fraction was then applied to DE-52 anion-exchange column chromatography according to the method of Kurokawa et al. (1990). Polypeptides shown in lane $\mathrm{d}$ were not adsorbed to the column. The materials eluted from the DE-52 column with $1 \mathrm{mM} \mathrm{Ca}^{2+}$ are shown in lane e. Although plasma and cytoplasmic gelsolin have been reported to elute from the column in the presence of $1 \mathrm{mM} \mathrm{Ca}^{2+}(\mathrm{Ku}-$ rokawa et al., 1990), the brain gelsolin was not eluted. By eluting the column with a buffer containing $1 \mathrm{M} \mathrm{NaCl}$, the myelin gelsolin was recovered. Following dialysis to remove the salt, the eluted fraction (lane f) was applied to blue Sepharose column 

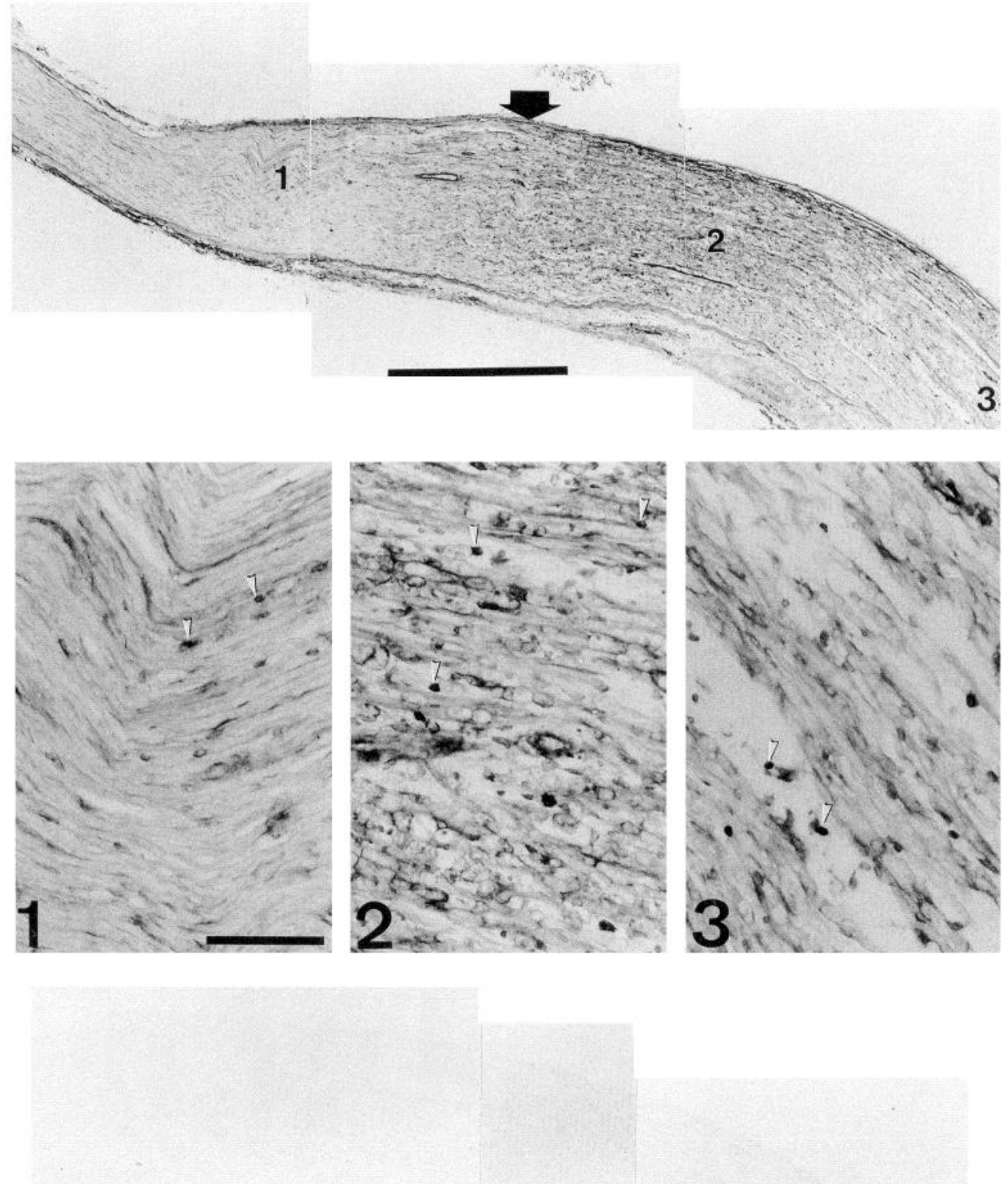

\section{Control}

Figure 8. Rat sciatic nerve injured by freezing. The nerve was taken out at 5 $\mathrm{d}$ after the injury, and was fixed and immunostained with the anti-gelsolin antibody using the $\mathrm{ABC}$ method. The upper panel shows the regenerating injured nerve. The arrow indicates the site of pressing with a steel stick precooled in liquid nitrogen. The intact or proximal side is on the left and the degenerated or peripheral side is on the right. Note the increased immunoreaction in the region around the area labeled 2. The numbers (1, 2, and 3) correspond to micrographs 1,2 , and 3 , which show almost intact, regenerating, and degenerated regions, respectively. Note the short Schwann cells in panel 2 that contain more gelsolin. Arrowheads indicate strongly immunoreactive macrophages. The lower panel shows the results of control study with normal rabbit IgG, and no specific immunostaining is observed. Scale bars: $1 \mathrm{~mm}$ (the upper and lower panel) and $100 \mu \mathrm{m}$ (the middle photomicrographs). chromatography. A major part of gelsolin, however, was not adsorbed to the column (lane g), in contrast to plasma and cytoplasmic gelsolin. Adsorbed gelsolin was eluted by $1 \mathrm{~mm}$ ATP (lane h). The eluted fraction $(2 \mathrm{ml})$ was mainly composed of gelsolin. Actin was not a contaminant of the purified gelsolin fraction, confirmed by dot immunoblotting using anti-actin polyclonal antibody (data not shown).

The severing and capping activity of the purified gelsolin was investigated (Figs. 13, 14). Monomeric or polymerized rabbit skeletal muscle actin was mixed with the gelsolin in the presence or absence of $\mathrm{Ca}^{2+}$. Following incubation, the apparent viscosity of the mixtures was measured. The gelsolin reduced viscosity of actin filaments only in the presence of $\mathrm{Ca}^{2+}$. When monomeric actin was polymerized in the presence of the gelsolin and $\mathrm{Ca}^{2+}$, viscosity was also reduced (Fig. 14). Under the same experimental condition, the human plasma gelsolin showed similar effects on actin (data not shown). Electron microscopy revealed that the myelin gelsolin fragmented actin filaments only in the presence of $\mathrm{Ca}^{2+}$ (Fig. 14).

\section{Discussion}

In the present study, we have investigated the gelsolin localization in the CNS and PNS employing immunohistochemical staining as well as biochemical procedure. The results indicate 

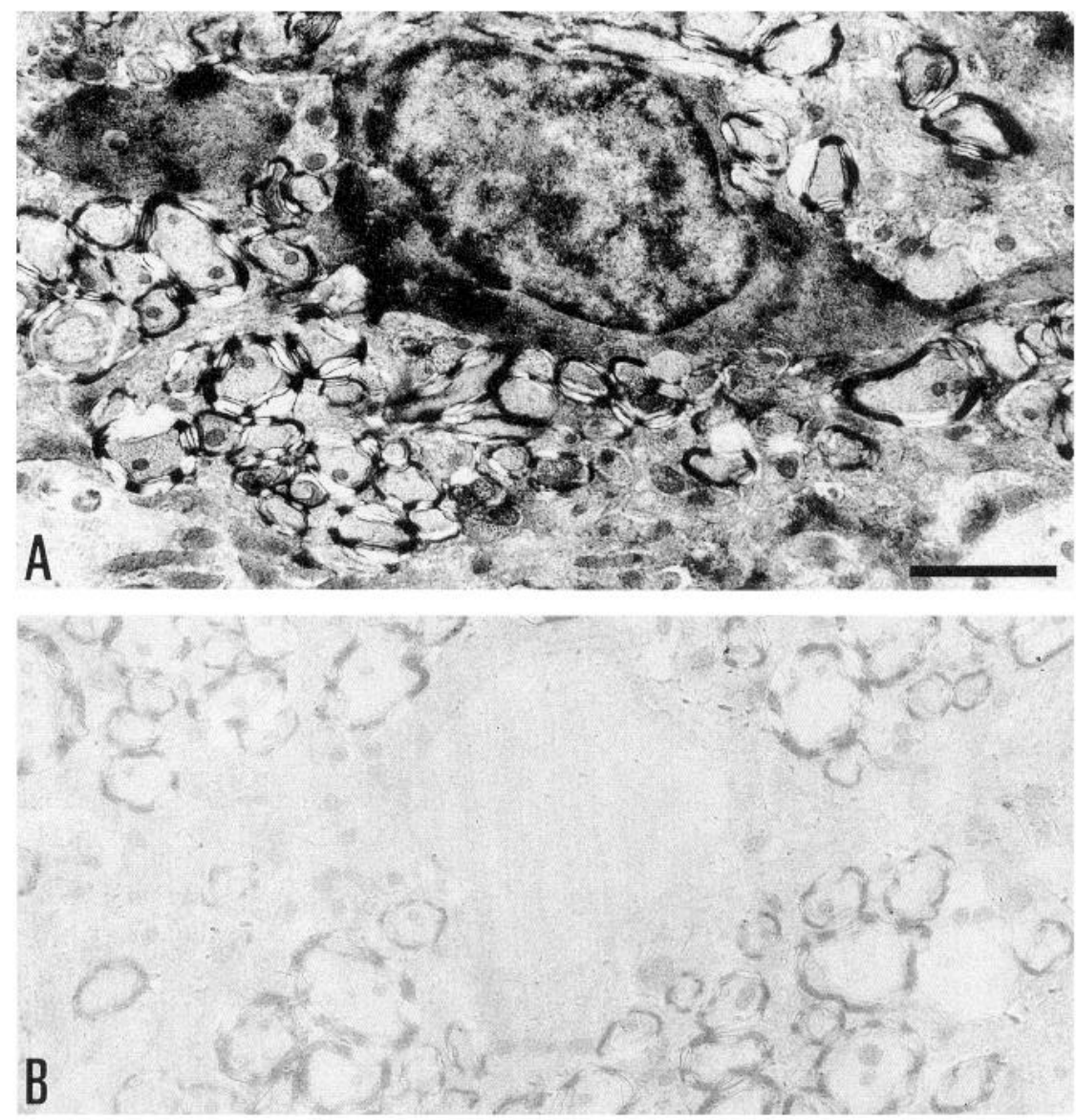

Figure 9. The localization of gelsolin in oligodendrocytes was examined by electron microscopy. The brain sections were prepared from the pons of a 21-d-old male rat. Following immunostaining using the $\mathrm{ABC}$ method, the sections were postfixed with $\mathrm{OsO}_{4}$, embedded in Epon, and stained with lead citrate. $A$ shows the interfascicular type of oligodendrocyte. Note the positive staining of myelin sheaths. The nuclear staining was observed in the brain sections fixed with glutaraldehyde. Control staining with normal rabbit IgG is shown in $B$; an interfascicular cell is not stained. $C$ shows the perineuronal satellite cell type of oligodendrocytes. $N$ indicates neurons. Scale bar, $2 \mu \mathrm{m}$.

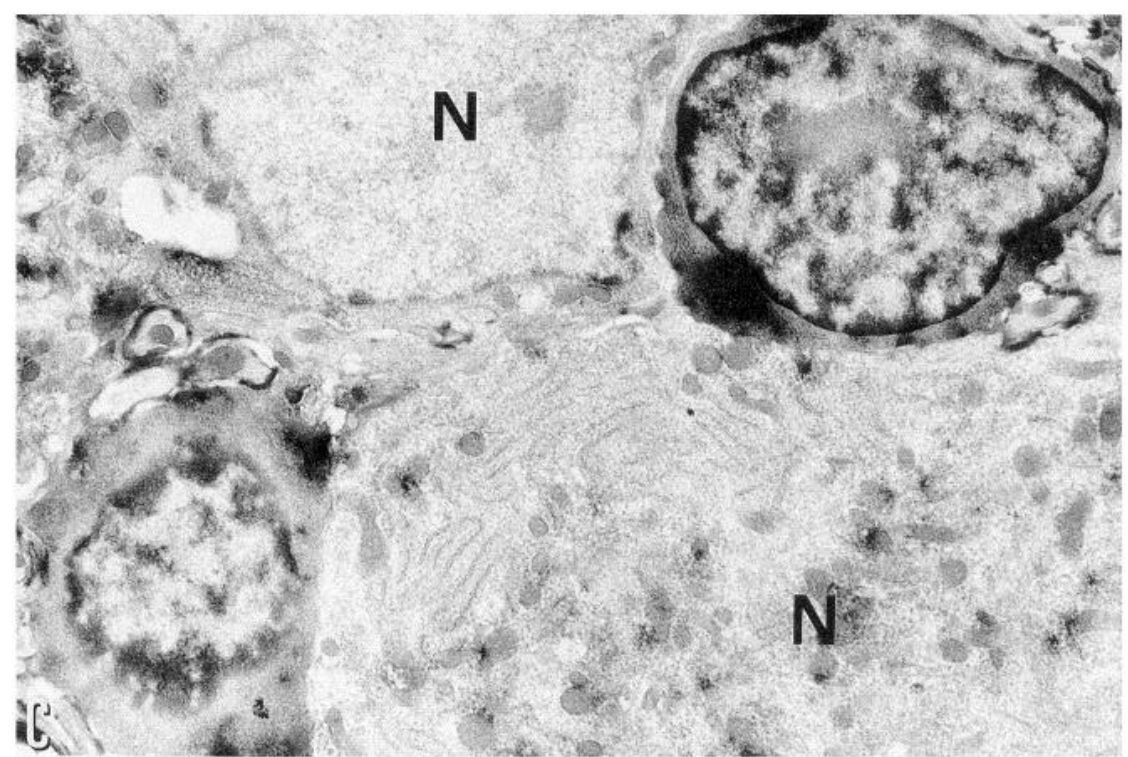

that gelsolin in the nervous system is specifically accumulated in the myelin-forming cells, which are oligodendrocytes and Schwann cells. No detectable immunostaining was observed in neurons and other classes of glial cells. The enrichment of gelsolin in oligodendrocytes in the CNS suggests that gelsolin can be used as a marker for the cells. Although many markers have been reported for myelin-forming cells such as galactocerebroside, cyclic nucleotide phosphohydrolase, and MBPs, a cyto- skeletal marker has not been well characterized. When using the anti-gelsolin antibody, it is advantageous to stain the whole body of oligodendrocytes in immature rat brain, including their wrapping processes around the neurites.

A small amount of gelsolin was present in the cerebral cortex at embryonic day 18 (data not shown), before oligodendrocytes differentiate from the oligodendrocyte-type 2 astrocyte (O-2A) progenitor cells (Miller et al., 1985). Moreover, gelsolin was 

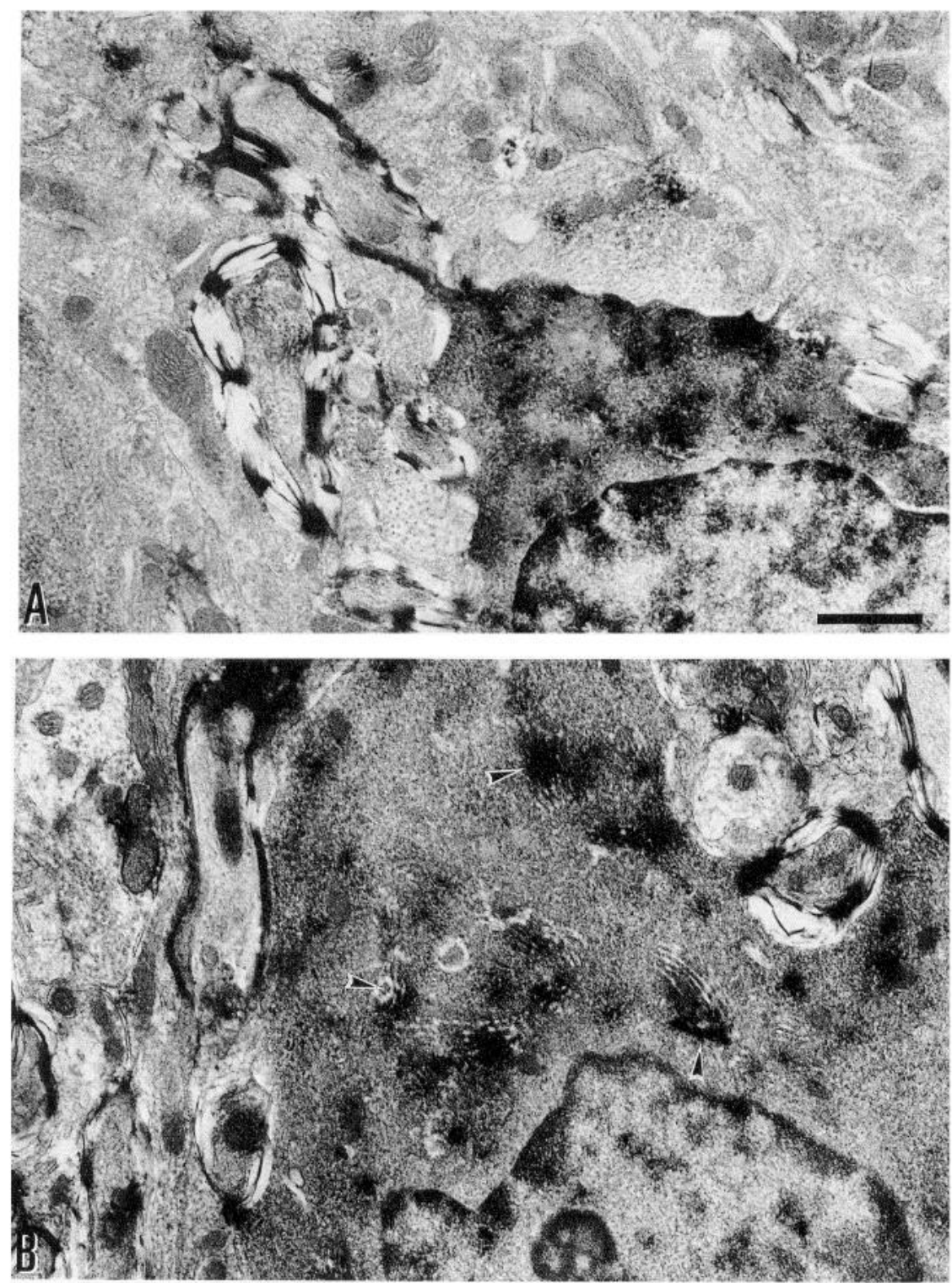

Figure 10. The oligodendrocyte process is shown in $A$, with high magnification. Gelsolin immunoreactivity is seen along the process projecting into the myelin sheath. $B$ shows strong immunoreaction around Golgi apparatus of oligodendrocytes (arrowheads). The brain sections were prepared from the pons of a 21-d-old male rat. Scale bar, $1 \mu \mathrm{m}$. hardly detected in oligodendrocytes even in postnatal brain before postnatal day $8-15$ (Fig. 6). This suggests that a small amount of gelsolin is derived from other cell types. Recently, we have identified gelsolin localization in growth cones of dorsal root ganglion neurons and PC12 cells differentiated with NGF, although the content is not high (Tanaka et al., unpublished observations). Therefore, some gelsolin in pre- and perinatal rat brain might be originated in neurons. As gelsolin localization in fibroblasts has been reported (Cooper et al., 1988), gelsolin might be present in non-neural cells. Their content of gelsolin, however, seems far lower than that in the myelin-forming cells. When the cells in the primary culture system of rat dorsal root ganglion are immunostained with anti-gelsolin antibody, the staining of fibroblasts is far weaker than that of Schwann cells (Tanaka et al., unpublished observation).

The localization of gelsolin in compact myelin was evidenced in the immunohistochemical and the subcellular fractionation study. Low concentration of detergents such as $0.1 \%$ Triton $\mathrm{X}-100$ could not solubilize gelsolin in the myelin fraction (data not shown), also suggesting that not only the soluble fraction around the Ranvier's nodes but myelin membrane itself contains gelsolin. This is an unusual distribution for gelsolin, considering the observation in non-neural cells that gelsolin near plasma membrane usually exists with actin filaments (Gavazzi et al., 1989; Hartwig et al., 1989). Gelsolin localization in compact myelin, however, might be reasonable, since myelin contains abundant polyphosphoinositide and enzymes related to phosphatidylinositol turnover (Saltiel et al., 1987), and gelsolin binds to polyphosphoinositide (Janmey et al., 1992). Indeed, the relationship between polyphosphoinositide and myelinogenesis has been suggested in developmental studies. In rat brain, the PIP kinase activity was elevated from day 13 after birth, when myelinogenesis began (Salway et al., 1968). Shaikh and Palmer $(1976,1977)$ reported that deposition of $\mathrm{PIP}_{2}$ correlated closely with myelinogenesis in chick brain and sciatic nerves. The polyphosphoinositide in the myelin membrane might regulate gelsolin function.

Gelsolin-like protein has been detected in nervous tissues 
Figure 11. The localization of gelsolin in compact myelin is shown using the immunogold method $(A)$. Normal rabbit IgG does not stain myelin membrane $(B)$. The sections shown here were stained with both lead citrate and uranyl acetate. The brain sections were prepared from the pons of a 21-d-old male rat. Scale bar, $200 \mathrm{~nm}$.
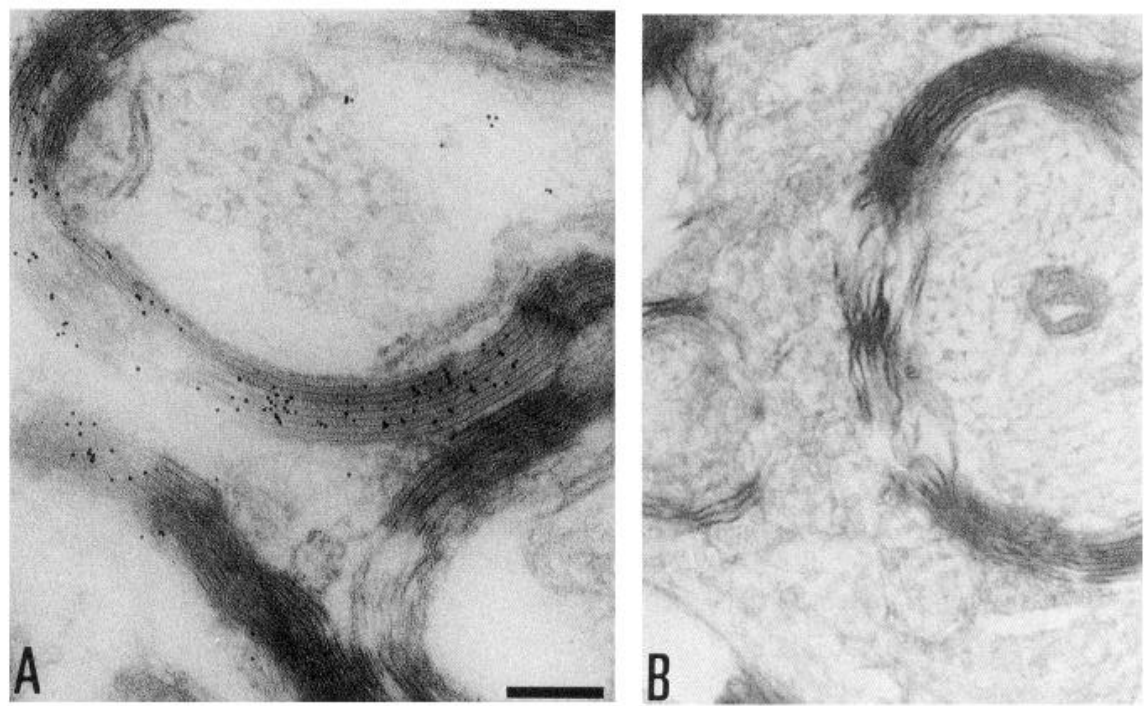

(Nishida et al., 1981; Yin et al., 1981; Verkhovsky et al., 1984). Complete purification, however, was difficult because the protein was tightly linked to monomeric actin. Since SDS was used to separate the two proteins (Petrucci et al., 1983), full characterization of brian gelsolin has been difficult. In the present study, however, the protein was easily purified from the myelin fraction using two column chromatographies without any contamination of actin. This might be related to the abundance of polyphosphoinositide in the myelin fraction; that is, the EGTAresistant actin-gelsolin complex might be dissociated by PIP or $\mathrm{PIP}_{2}$ (Janmey et al., 1987).

The myelin gelsolin is not only a capping protein, as has been suggested (Isenberg et al., 1983), but also it can sever actin filament. Although the $\mathrm{Ca}^{2+}$-dependent severing activity was similar to that of cytoplasmic or plasma gelsolin, it has not been elucidated whether the protein is the same as other gelsolin. The molecular weight of the brain gelsolin was not distinguishable from that of gelsolin in other tissues such as lung or stomach (data not shown). The behavior of the gelsolin against the column chromatographies, however, was different. In particular, it seems interesting that the protein could not be eluted from the DE-52 column by the $\mathrm{Ca}^{2+}$ buffer, although the plasma and cytoplasmic gelsolin were eluted under the same condition $(\mathrm{Ku}-$ rokawa et al., 1990). The remarkable accumulation in the myelin membrane might suggest its difference from gelsolin in nonneural cells, considering that gelsolin has been purified from soluble fraction of macrophages. The primary structure should be determined to elucidate whether the brain gelsolin is the same as the cytoplasmic gelsolin. Since the brain gelsolin can be purified from the myelin fraction of bovine spinal cord using a method similar to the one described here (data not shown), we are going to compare the properties of the protein with those of bovine plasma gelsolin.

The brain gelsolin might be involved in myelinogenesis, in view of several present findings, including its enrichment in myelin-forming cells and its elevated expression during myelinogenesis and remyelination. The severing, capping, and nucleating activity of gelsolin might be important for protrusive movements of cells (Oster and Perelson, 1987), because the protein can alter the intracellular osmotic pressure by remodeling the actin networks with gelation factors such as actinbinding protein (ABP or filamin) (Ito et al., 1992). During my-
Figure 12. The brain gelsolin was purified from the myelin fraction as shown here. $A$ is silver-stained SDS- $10 \%$ polyacrylamide gel, and $B$ is the corresponding immunoreplica with the anti-gelsolin antibody. Lane $a$, the myelin fraction prepared by the method of axonal flotation; lane $b$, solubilized proteins by $1 \%$ deoxycholic acid; lane $c$, the fraction applied to DE-52 column after removing the detergent by dialysis; lane $d$, flowthrough fraction from DE-52 column; lane $e$, eluate from the column by $1 \mathrm{~mm} \mathrm{CaCl}$; lane $f$, eluate from the column by $1 \mathrm{M} \mathrm{NaCl}$, which was applied to blue Sepharose column after removing the salt by dialysis; lane $g$, flowthrough fraction from blue Sepharose column; lane $h$, eluate from blue Sepharose column by $1 \mathrm{~mm}$ ATP. The fraction of lane $h$ was used for the following biochemical assays.

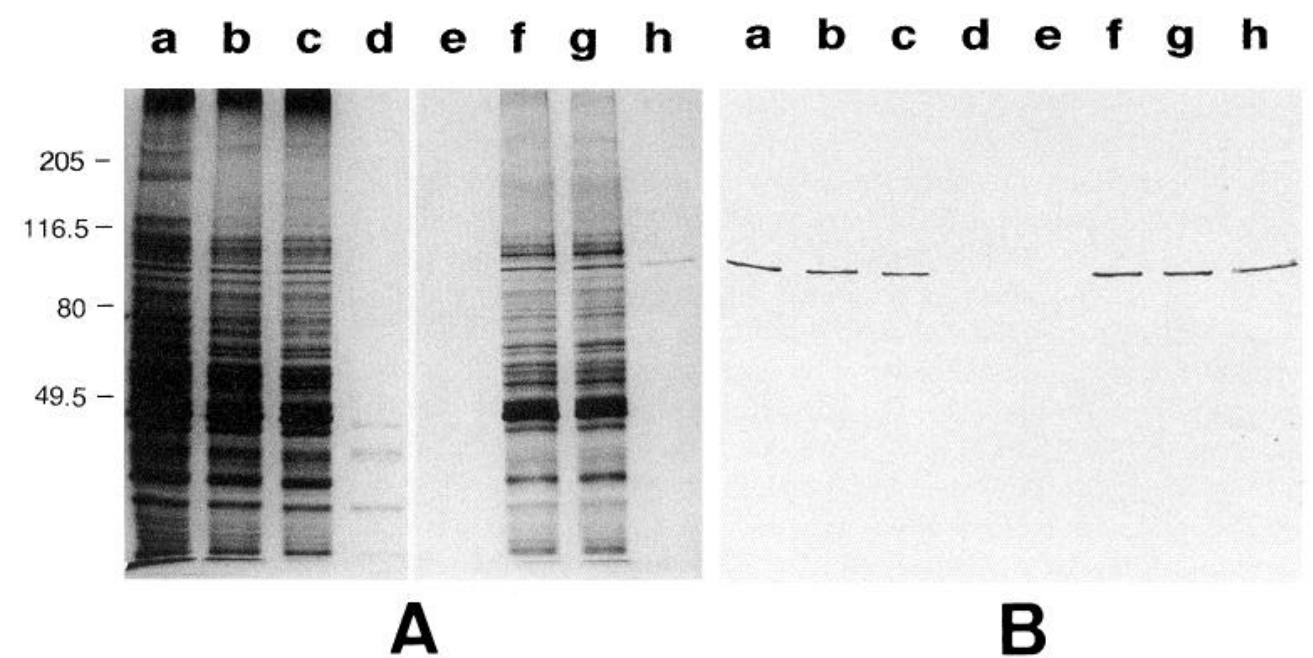




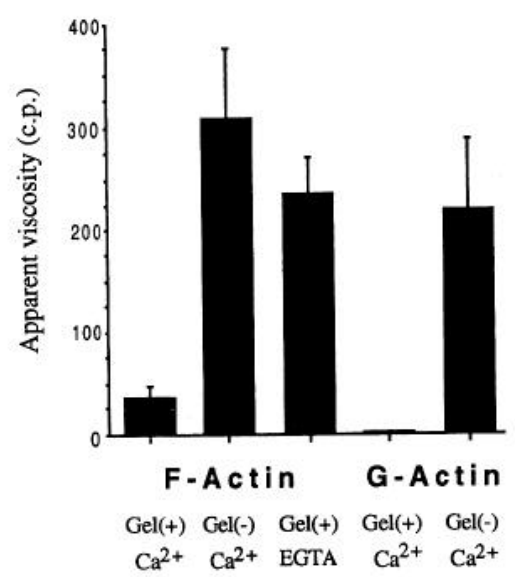

Figure 13. Effects of the brain gelsolin on actin were evaluated by falling ball viscometry. The apparent viscosity of actin filaments $(F$ Actin) incubated with or without gelsolin and $\mathrm{Ca}^{2+}$, and the viscosity of the mixture are shown in the three columns to the left. Monomeric actin $(G-A c t i n)$ was polymerized in the presence of $\mathrm{Ca}^{2+}$ with or without gelsolin and viscosity of the mixture is shown in the two columns to the right. The apparent viscosity is expressed along the y-axis (centipoise, c.p.) with averaged value and standard error bar calculated from the data of six experiments. The assay conditions are described in Materials and Methods.

elin formation, the wrapping movement of oligodendrocytes and Schwann cells around the neurites might be regarded as protrusive activity of their lamellipodia. Gelsolin might participate in these wrapping movements. The remarkable accumulation of gelsolin at Ranvier's nodes suggests its involvement in the longitudinal movements of the lamellipodia along the neurites. Since gelsolin localizes in rapidly moving cell organs such as ruffling membranes of fibroblasts (Cooper et al., 1988) or podosomes of $s r c$-transformed cells (Gavazzi et al., 1989), the wrapping movement of the myelin-forming cells might also be a rapid phenomenon. Considering the presence of gelsolin in the satellite cell type of oligodendrocytes and in the cells of old rat brain, though the content seems lower, other roles of gelsolin might be suspected. Further investigation is required to clarify this point.

\section{References}

Adams CWM, Bayliss OB, Hallpike JF, Turner DR (1971) Histochemistry of myelin-XII. Anionic staining of myelin basic proteins for histology, electrophoresis and electron microscopy. J Neurochem 18:389-394.

Ashino N, Sobue K, Seino Y, Yabuuchi H (1987) Purification of an $80 \mathrm{kDa} \mathrm{Ca}^{2+}$-dependent actin-modulating protein, which severs actin filaments, from bovine adrenal medulla. J Biochem 101:609-617.

Aveldaño MI, Dîaz RS, Rugueiro P, Monreal J (1991) Solubilization of myelin membranes by detergents. J Neurochem 57:250-257.

Bray D, White JG (1988) Cortical flow in animal cells. Science 239: 883-888.

Chen W-T (1989) Proteolytic activity of specialized surface protrusions formed at rosette contact sites of transformed cells. J Exp Zool 251:167-185.

Cooper JA, Loftus DJ, Frieden C, Bryan J, Elson EL (1988) Localization and mobility of gelsolin in cells. J Cell Biol 106:1229-1240.

Cunningham CC, Stossel TP, Kwiatkowski DJ (1991) Enhanced motility in NIH 3T3 fibroblasts that overexpress gelsolin. Science 251: 1233-1236.

De Lozanne A, Spudich JA (1987) Disruption of the Dictyostelium myosin heavy chain gene by homologous recombination. Science 236: 1086-1091.

Gavazzi I, Nermut M, Marchisio PC (1989) Ultrastructure and goldimmunolabeling of cell-substratum adhesions (podosomes) in RSVtransformed cells. J Cell Sci 94:85-99.

Hartwig JH (1992) Mechanism of actin rearrangements mediating platelet activation. J Cell Biol 118:1421-1422.

Hartwig JH, Chambers KA, Stossel TP (1989) Association of gelsolin with actin filaments and cell membranes of macrophages and platelets. J Cell Biol 108:467-479.

Hsu S-M, Raine L, Fanger H (1981) Use of avidin-biotin-peroxidase complex $(\mathrm{ABC})$ in immunoperoxidase techniques. J Histochem $\mathrm{Cy}-$ tochem 29:577-580.

Isenberg G, Ohnheiser R, Maruta H (1983) 'Cap 90', a 90-kDa Ca ${ }^{2+}$. dependent $\mathrm{F}$-actin-capping protein from vertebrate brain. FEBS Lett 163:225-229

Ito T, Suzuki A, Stossel TP (1992) Regulation of water flow by actinbinding protein-induced actin gelation. Biophys J 61:1301-1305.

Janmey PA, Stossel TP (1987) Modulation of gelsolin function by phosphatidylinositol 4,5-bisphosphate. Nature 325:362-364.

Janmey PA, Iida K, Yin HL, Stossel TP (1987) Polyphosphoinositide micelles and polyphosphoinositide-containing vesicles dissociate endogenous gelsolin-actin complexes and promote actin assembly from the fast growing end of actin filaments blocked by gelsolin. J Biol Chem 262:12228-12236.

Janmey PA, Lamb J, Allen PG, Matsudaira PT (1992) Phosphoinositide-binding peptides derived from the sequence of gelsolin and villin. J Biol Chem 267:11818-11823.
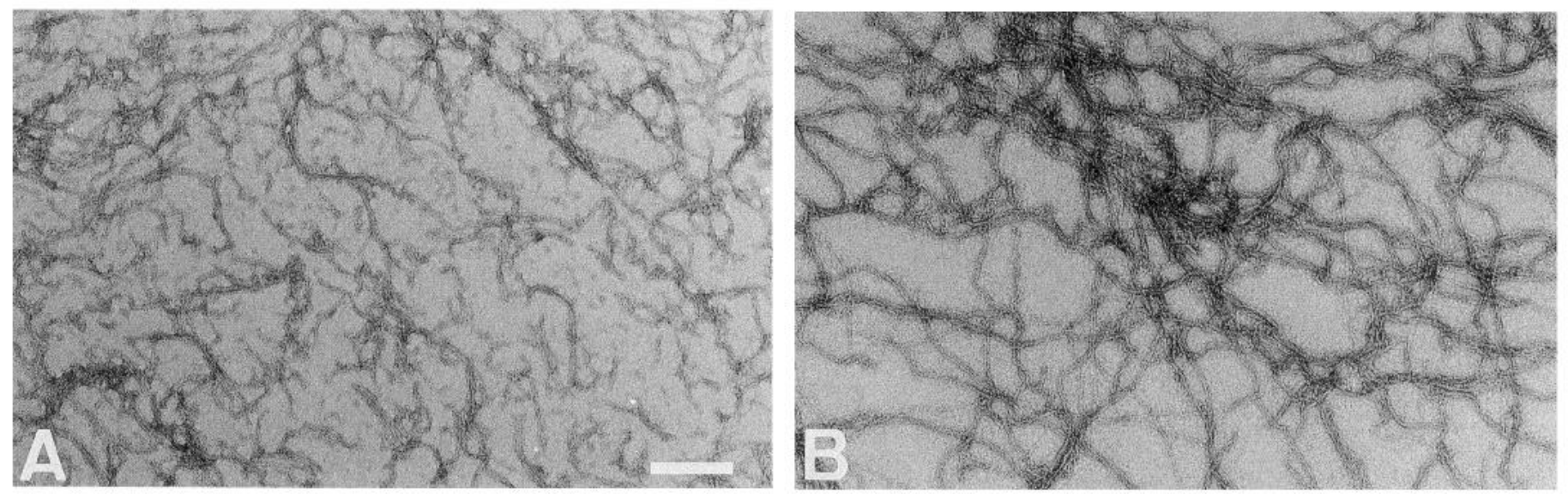

Figure 14. Severing activity of the brain gelsolin was examined by electron microscopy. The gelsolin and actin filaments with or without $\mathrm{Ca}^{2+}$ were mixed and incubated. The mixtures were then negatively stained and viewed by electron microscopy. $A$ shows the fragmented actin filaments in the presence of $\mathrm{Ca}^{2+}$. Without $\mathrm{Ca}^{2+}$, actin filaments seem intact in the presence of gelsolin $(B)$. Scale bar, $200 \mathrm{~nm}$. 
Kachar B, Behar T, Dubois-Dalcq M (1986) Cell shape and motility of oligodendrocytes cultured without neurons. Cell Tissue Res 244: 27-38.

Knecht DA, Loomis WF (1987) Antisense RNA inactivation of myosin heavy chain gene expression in Dictyostelium discoideum. Science 236:1081-1086.

Kurokawa H, Fujii W, Ohmi K, Sakurai T, Nonomura Y (1990) Simple and rapid purification of brevin. Biochem Biophys Res Commun 168:451-457.

Kwiatkowski DJ, Janmey PA, Mole JE, Yin HL (1985) Isolation and properties of two actin-binding domains in gelsolin. J Biol Chem 260: $15232-15238$

Kwiatkowski DJ, Stossel TP, Orkin SH, Mole JE, Colten HR, Yin HL (1986) Plasma and cytoplasmic gelsolins are encoded by a single gene and contain a duplicated actin-binding domain. Nature 323:455-458.

Laemmli UK (1970) Cleavage of structural proteins during the assembly of the head of bacteriophage T4. Nature 227:680-685.

Legrand C, Ferraz C, Clavel M-C, Rabié A (1986) Immunocytochemical localisation of gelsolin in oligodendroglia of the developing rabbit central nervous system. Dev Brain Res 30:231-235.

Legrand C, Ferraz C, Clavel M-C, Rabié A (1991) Distribution of gelsolin in the retina of the developing rabbit. Cell Tissue Res 264: 335-338.

LeVine SM, Macklin WB (1988) Biotin enrichment in oligodendrocytes in the rat brain. Brain Res 444:199-203.

Liem RKH, Yen S-H, Salomon GD, Shelanski ML (1978) Intermediate filaments in nervous tissues. J Cell Biol 79:637-645.

Miller RH, David S, Patel R, Abney ER, Raff MC (1985) A quantitative immunohistochemical study of macroglial cell development in the rat optic nerve: in vivo evidence for two distinct astrocyte lineages. Dev Biol 111:35-41.

Nishida E, Kuwaki T, Maekawa S, Sakai H (1981) A new regulatory protein that affects the state of actin polymerization. J Biochem 89: $1655-1658$.

Norton WT, Poduslo SE (1973) Myelination in rat brain: method of myelin isolation. J Neurochem 21:749-757.

Ostcr GF, Pcrelson AS (1987) The physics of cell motility. J Cell Sci [Suppl] 8:35-54.

Pardee JD, Spudich JA (1982) Purification of muscle actin. Methods Enzymol 85B:164-181.

Petrucci TC, Thomas C, Bray D (1983) Isolation of a $\mathrm{Ca}^{2+}$-dependent actin-fragmenting protein from brain, spinal cord, and cultured neurones. J Neurochem 40:1507-1516.

Raine CS (1989) Neurocellular anatomy. In: Basic neurochem, 4th ed (Siegel et al., eds), pp 3-33. New York: Raven.

Rodriguez del Castillo A, Lemaire S, Tchakarov I, Jeyapragasan M, Doucet J-P, Vitale ML, Trifaro J-M (1990) Chromaffin cell scinderin, a novel calcium-dependent actin filament-severing protein. EMBO J 9:43-52.

Sakurai T, Ohmi K, Kurokawa H, Nonomura Y (1990) Distribution of a gelsolin-like 74,000 mol. wt protein in neural and endocrine tissues. Neuroscience 38:743-756.

Saltiel AR, Fox JA, Sherline P, Sahyoun N, Cuatrecasas P (1987) Purification of phosphatidylinositol kinase from bovine brain myelin Biochem J 241:759-763.

Salway JG, Harwood JL, Kai M, White GL, Hawthorne JN (1968) Enzymes of phosphoinositide metabolism during rat brain development. J Neurochem 15:221-226.

Shaikh NA, Palmcr FBS T $_{\mathrm{T}}$ (1976) Deposition of lipids in the developing central and peripheral nervous system of the chicken. J Neurochem 26:597-603.

Shaikh NA, Palmer FBS ${ }_{\mathrm{T}} \mathrm{C}$ (1977) Phosphoinositide kinases in chick brain and sciatic nerve, a developmental study. J Neurochem 28:395402 .

Sobue K, Kanda K (1989) $\alpha$-Actinins, calspectin (brain spectrin or fodrin), and actin participate in adhesion and movement of growth cones. Neuron 3:311-319.

Sobue K, Fujio Y, Kanda K (1988) Tumor promoter induces reorganization of actin filaments and calspectin (fodrin or non-erythroid spectrin) in 3T3 cells. Proc Natl Acad Sci USA 85:482-486.

Stossel TP (1989) From signal to pseudopod. J Biol Chem 264:1826118264.

Symons MH, Mitchison TJ (1991) Control of actin polymerization in live and permeabilized fibroblasts. J Cell Biol 114:503-513.

Tanaka J, Watanabe T, Nakamura N, Sobue K (1993) Morphological and biochemical analyses of contractile proteins (actin, myosin, caldesmon and tropomyosin) in normal and transformed cells. J Cell Sci 104:595-606.

Tashiro T, Komiya Y (1991) Changes in organization and axonal transport of cytoskeletal proteins during regeneration. J Neurochem $56: 1557-1563$.

Verkhovsky AB, Surgucheva IG, Gelfand VI (1984) Phalloidin and tropomyosin do not prevent actin filament shortening by the $90 \mathrm{kD}$ protein-actin complex from brain. Biochem Biophys Res Commun 123:596-603.

Verkhovsky AB, Surgucheva IG, Svitkina TM, Tint IS, Gelfand VI (1987) Organization of stress fibers in cultured fibroblasts after extraction of actin with bovine brain gelsolin-like protein. Exp Cell Res $173: 244-255$

Yamamoto H, Terabayashi M, Egawa T, Hayashi E, Nakamura $H$, Kishimoto S (1989) Affinity separation of human plasma gelsolin on Affi-gel blue. J Biochem 105:799-802.

Yin HL, Stossel TP (1979) Control of cytoplasmic actin gel-sol transformation by gelsolin, a calcium-dependent regulatory protein. Nature 281:583-586.

Yin HL, Albrecht JH, Fattoum A (1981) Identification of gelsolin, a $\mathrm{Ca}^{2+}$-dependent regulatory protein of actin gel-sol transformation, and its intracellular distribution in a variety of cells and tissues. I Cell Biol 91:901-906. 